https://doi.org/10.52837/27382702-2021-34.2-10

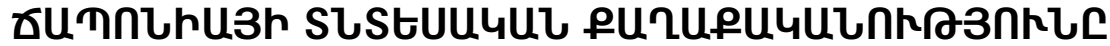 cusuusulnhU (1992-2021 pр.)*
}

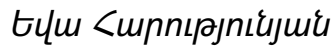

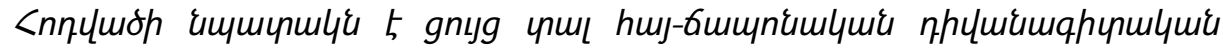

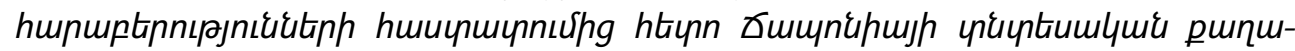

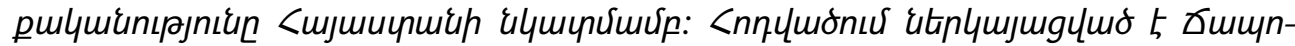

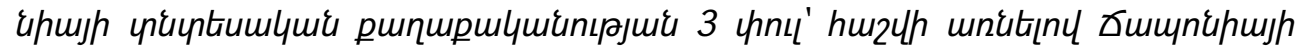

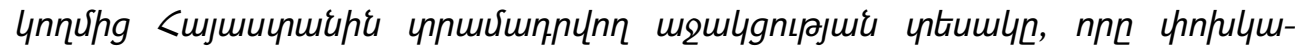

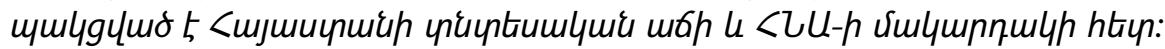

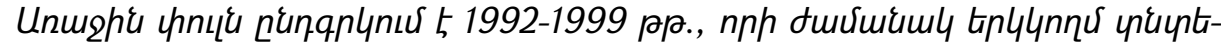

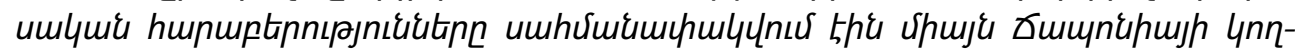

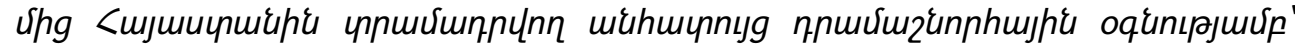

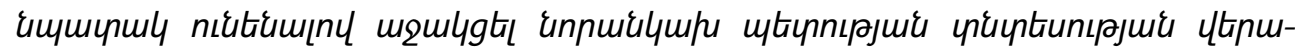

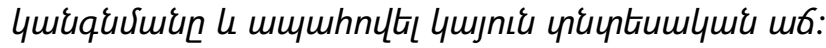

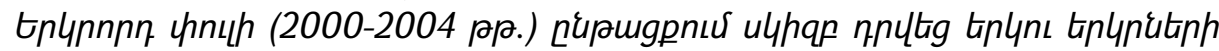

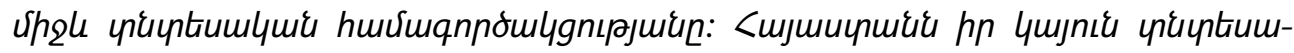

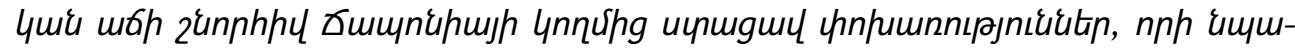

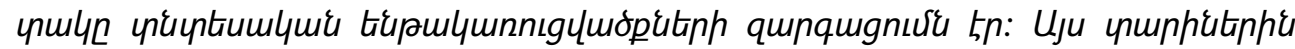

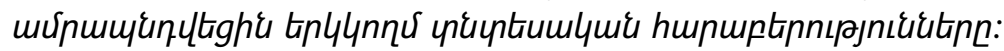

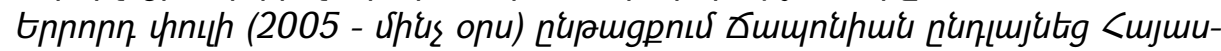

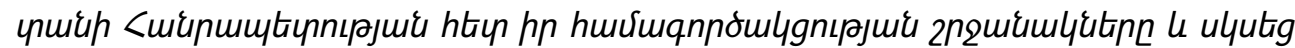

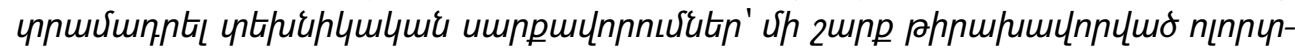

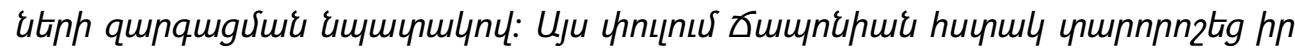

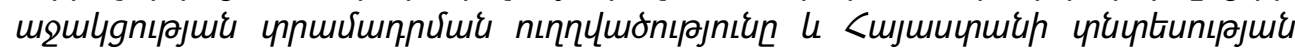

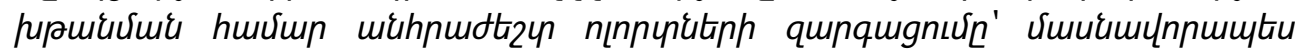

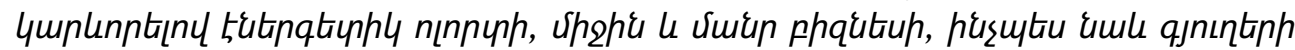

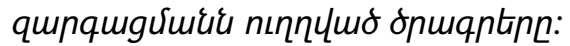

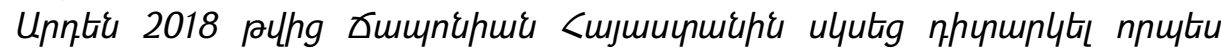

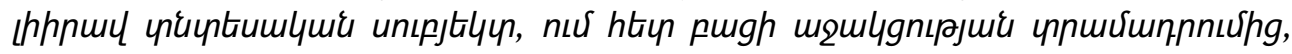

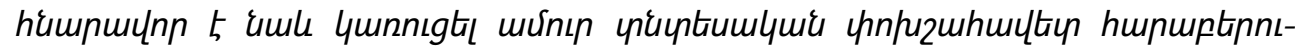

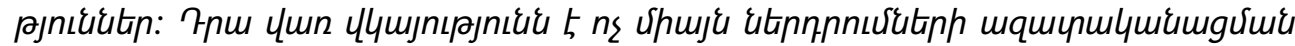

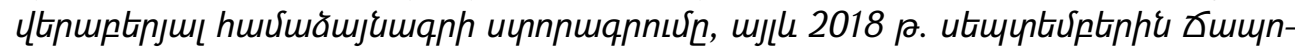

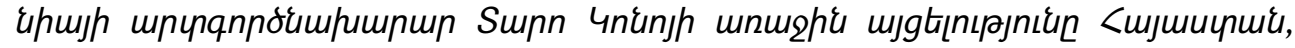

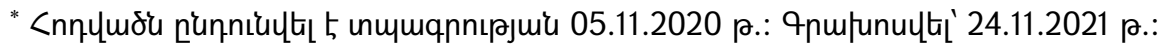




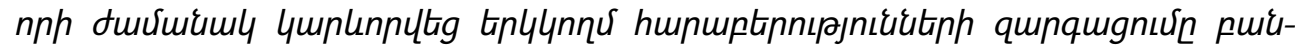

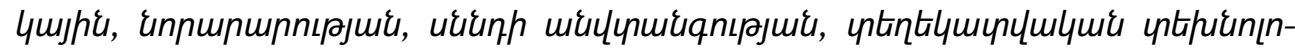

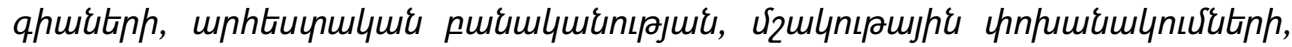

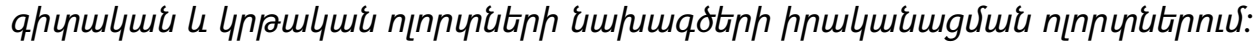

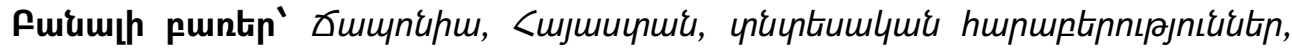

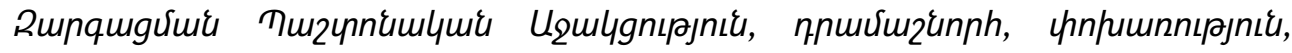

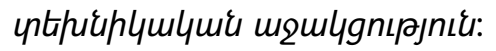

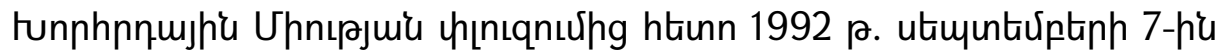

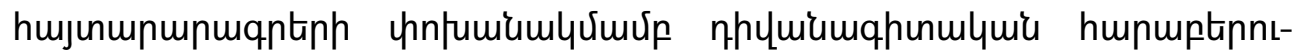

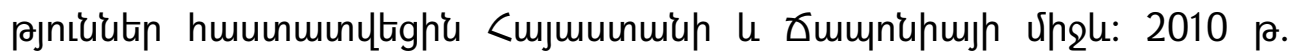

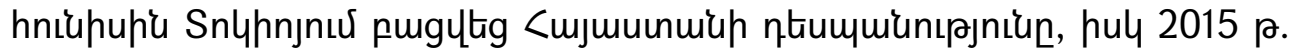

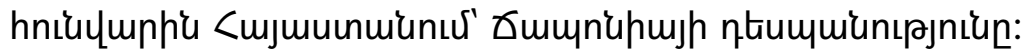

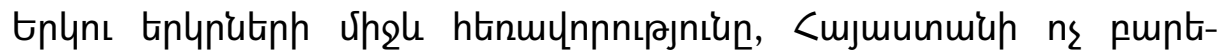
umumuin m2łumphuqnulymi

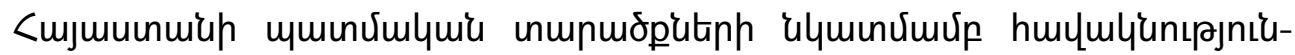

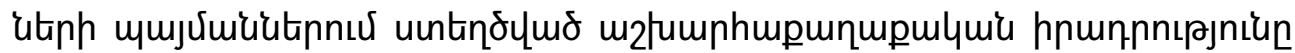

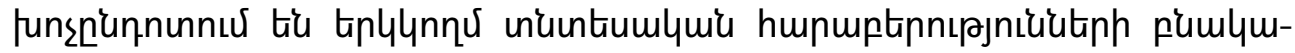

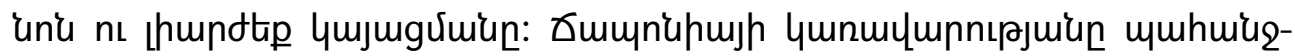

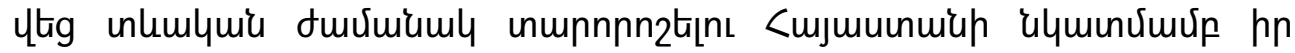

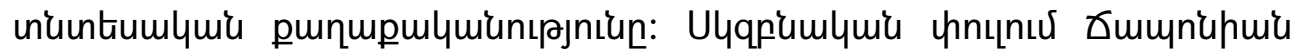

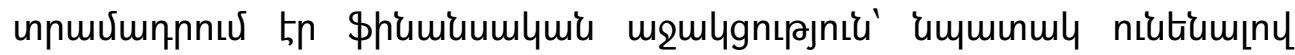

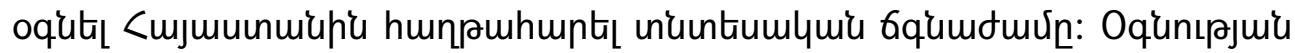

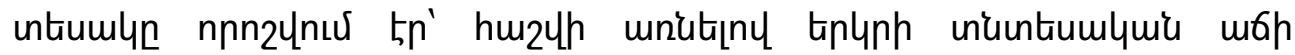

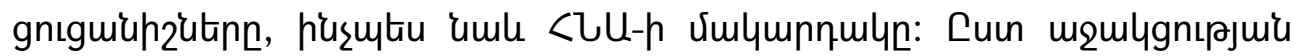

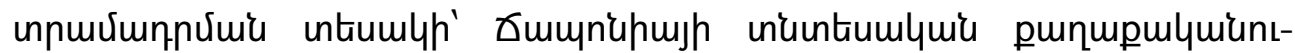

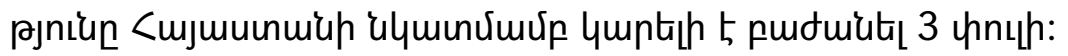

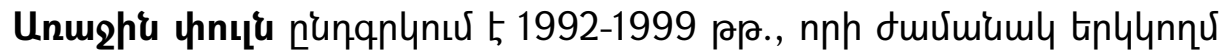

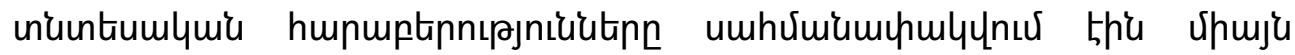

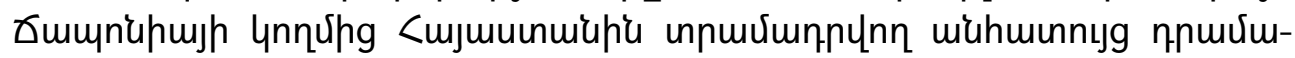

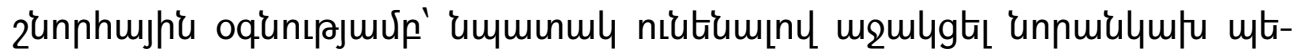

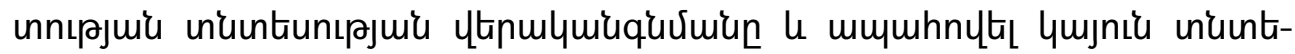
umluitu m6:

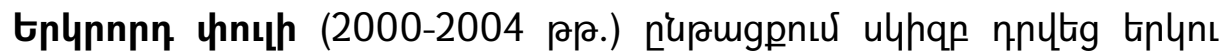

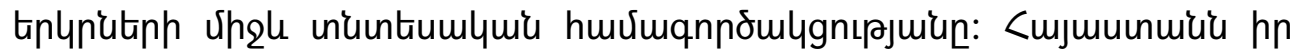

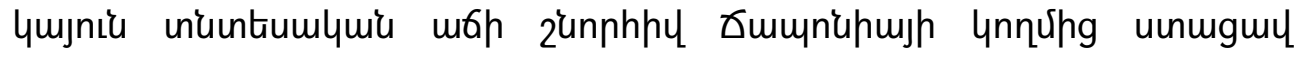




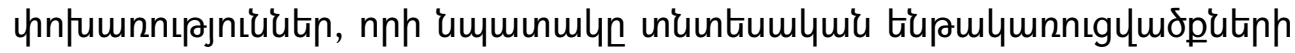

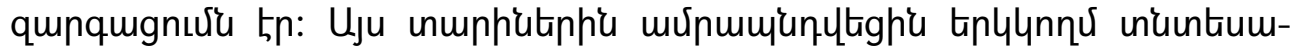
чuiu hunuptinnıpjniüutinn:

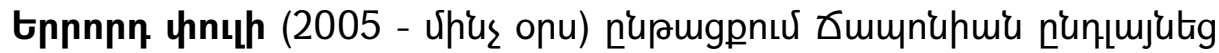

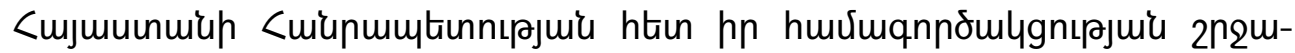

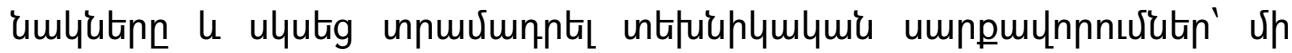

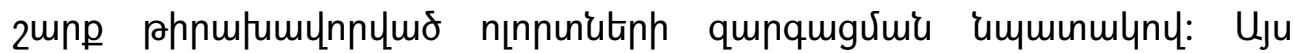

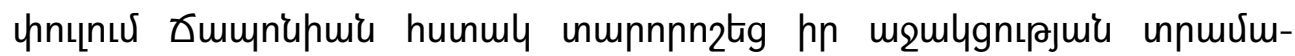

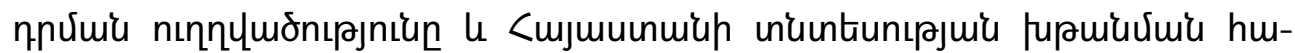

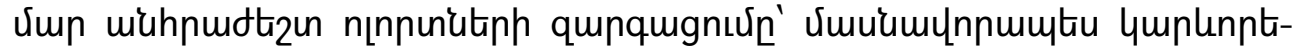

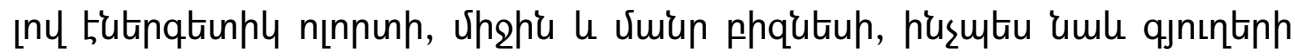

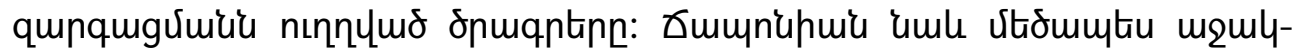

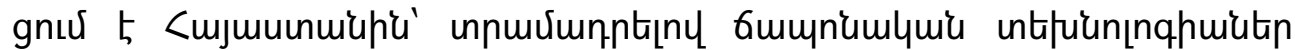

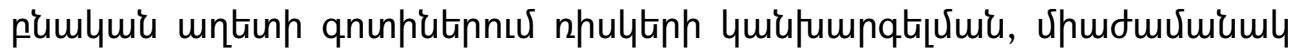

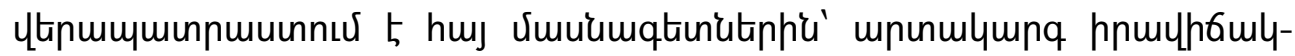

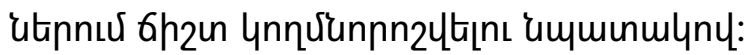

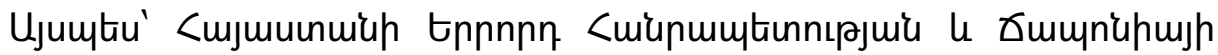

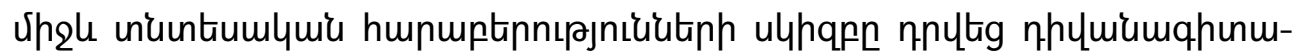

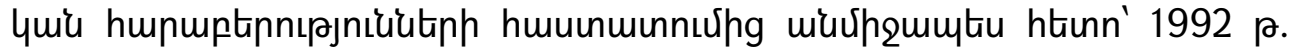

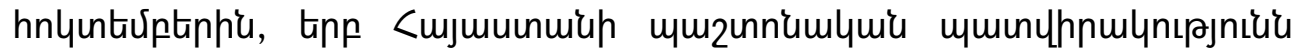

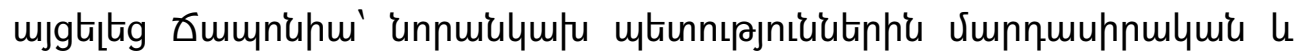

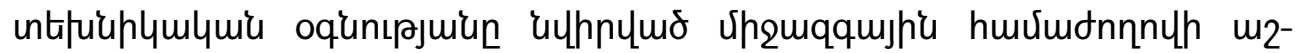

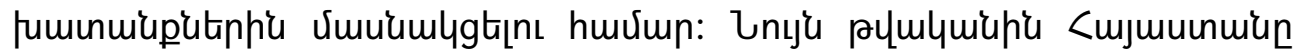

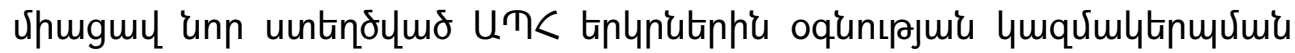

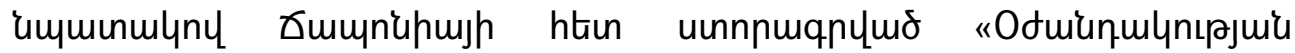

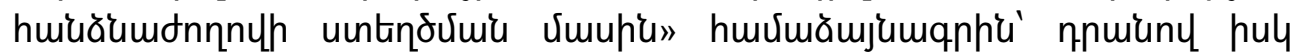

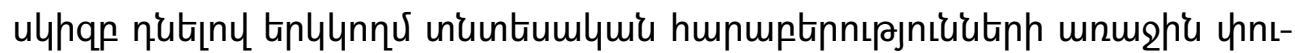

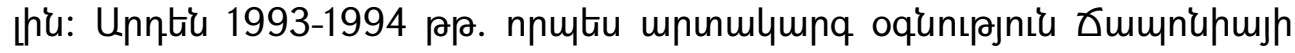

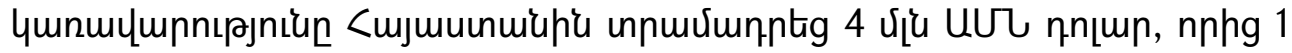

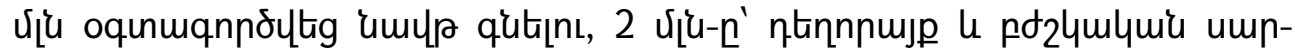

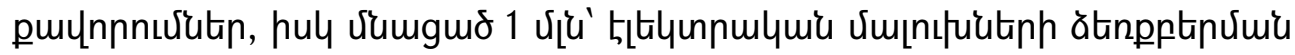

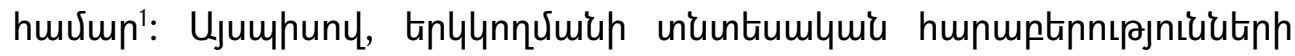

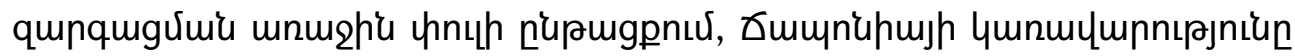

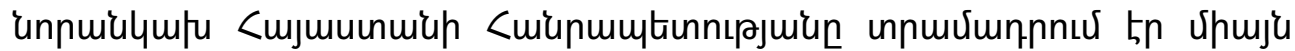

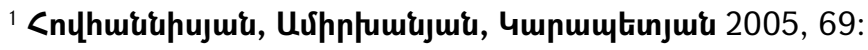




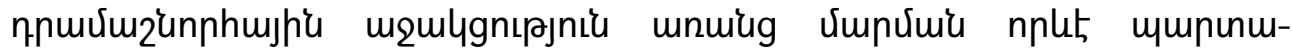

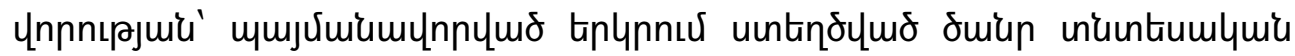

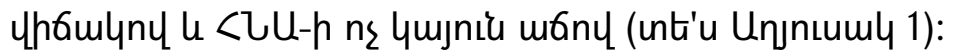

\section{Unjnsuml 1}

\begin{tabular}{|c|c|c|c|c|c|c|c|c|c|}
\hline \multicolumn{10}{|c|}{ 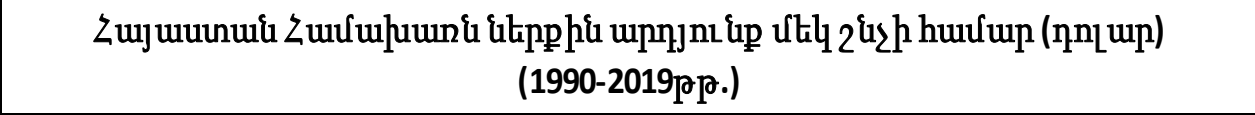 } \\
\hline 1990 & 1991 & 1992 & 1993 & 1994 & 1995 & 1996 & 1997 & 1998 & 1999 \\
\hline$\$ 651$ & $\$ 600$ & $\$ 364$ & $\$ 348$ & $\$ 383$ & $\$$ & $\$ 538$ & $\$ 558$ & \begin{tabular}{|l|l}
$\$ 649$ & \\
\end{tabular} & $\$ 637$ \\
\hline 2000 & 2001 & 2002 & 2003 & 2004 & 2005 & 2006 & 2007 & 2008 & 2009 \\
\hline$\$ 664$ & $\$ 741$ & $\$ 835$ & $\$ 992$ & $\$ 1271$ & $\$ 1753$ & $\$ 2301$ & $\$ 3347$ & $\$ 4276$ & $\$ 3193$ \\
\hline 2010 & 2011 & 2012 & 2013 & 2014 & 2015 & 2016 & 2017 & 2018 & 2019 \\
\hline 3432 & $\$ 3761$ & $\$ 3685$ & $\$ 3844$ & $\$ 3995$ & $\$ 3618$ & $\$ 3606$ & $\$ 3934$ & $\$ 4237$ & $\$ 4623$ \\
\hline
\end{tabular}

Unрјnıр' ВВП Армении, 1990-2019, https://be5.biz/makroekonomika/gdp/am.html

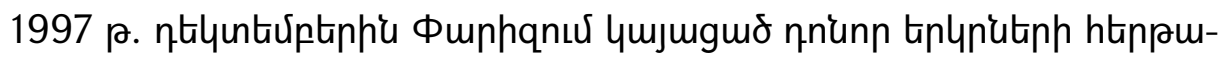

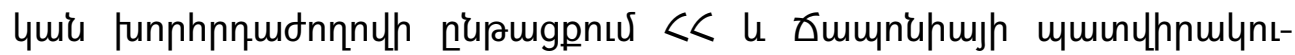

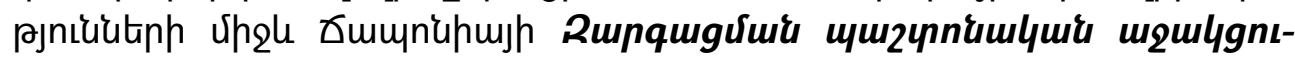

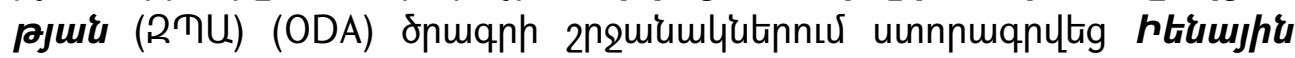

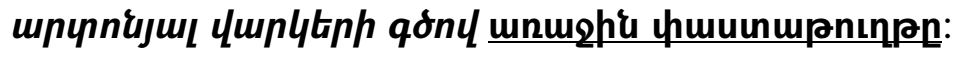

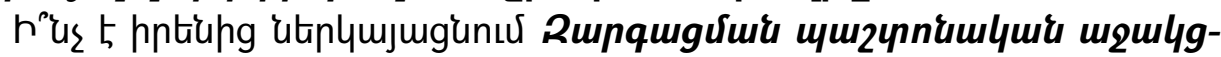
sur dnmqhnn:

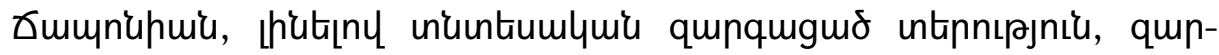

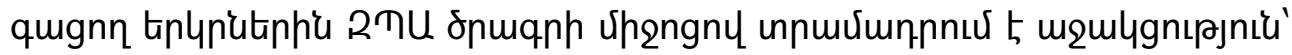

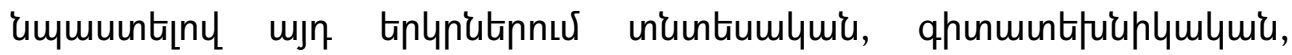

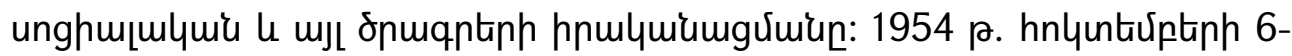

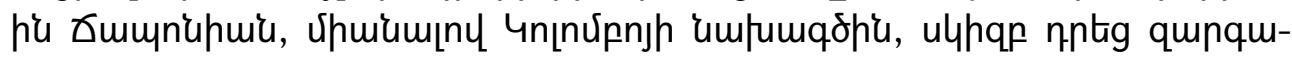

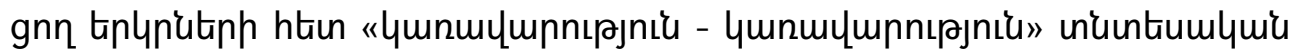

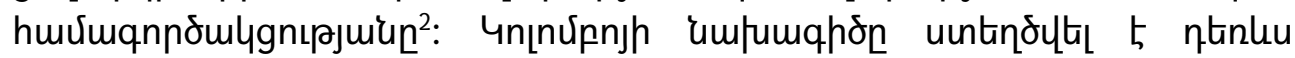

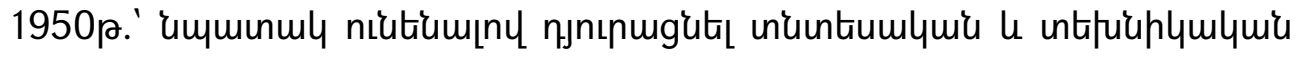

\footnotetext{
2 Japan's ODA-Fifty Years in the Front Lines, Website of Japan International Cooperation Agency, https://www.jica.go.jp/english/publications/reports/network/archive_2004/vol_25_2.html
} 


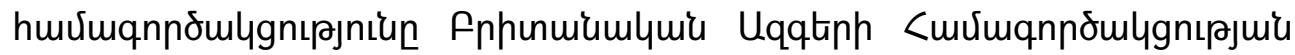

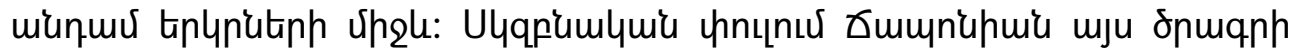

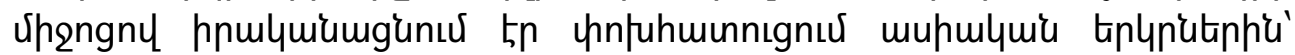

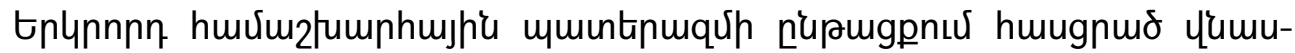

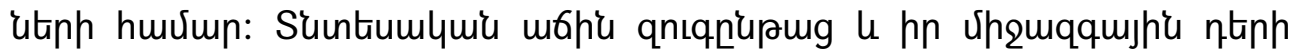

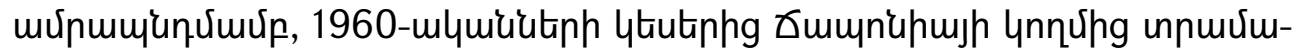

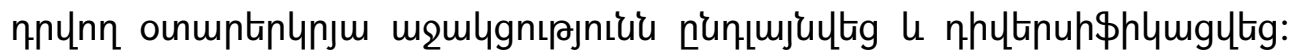

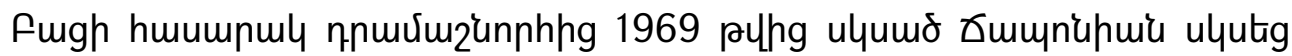

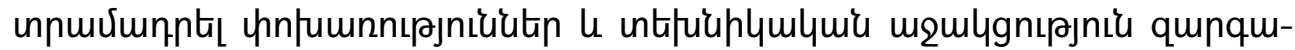
gnn tplunutiphi:

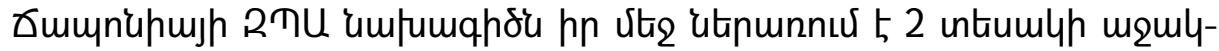

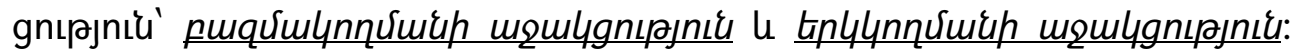

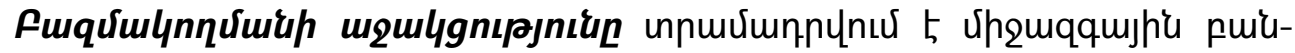

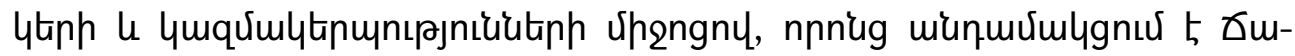

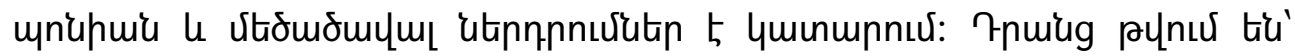

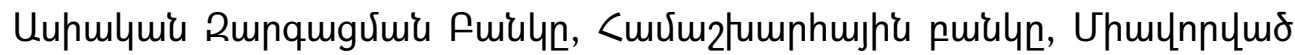

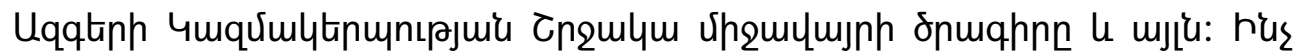

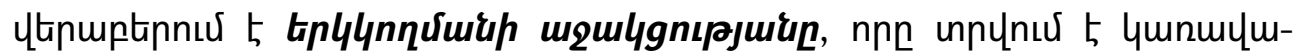

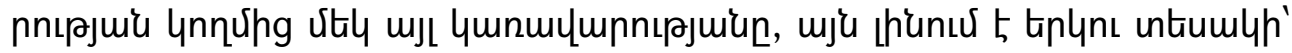

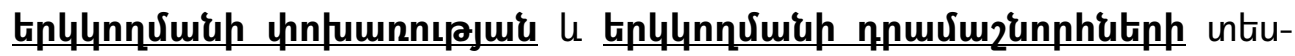

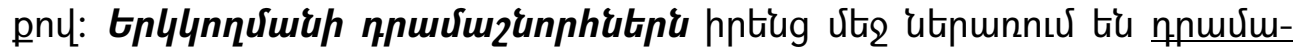

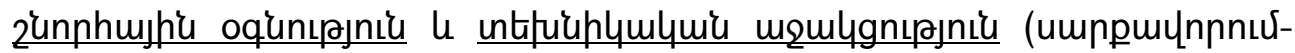

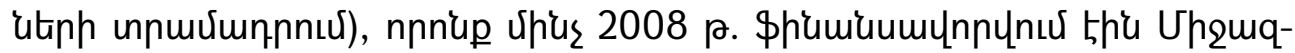

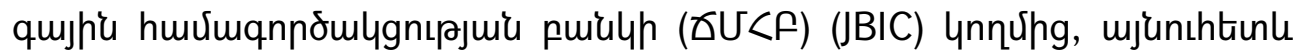

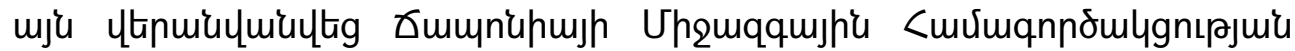

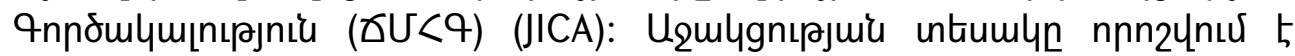

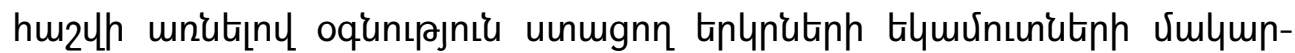

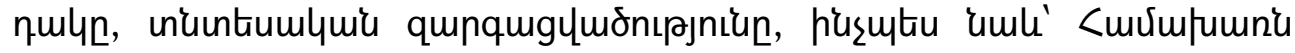

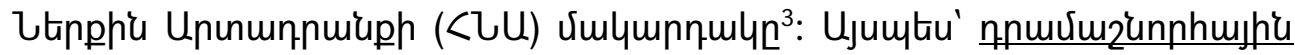

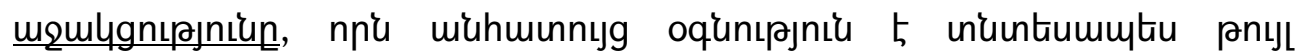

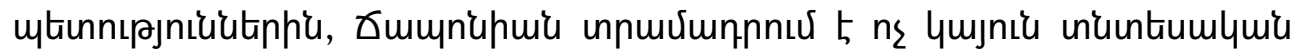

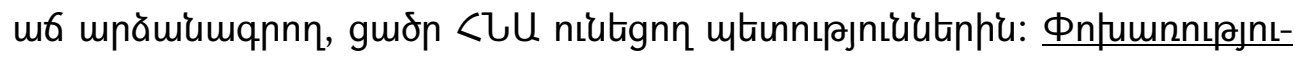

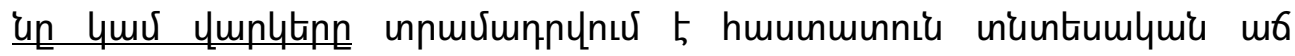

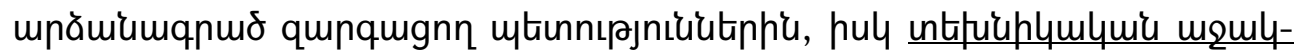

\footnotetext{
${ }^{3}$ ODA by Region Central Asia and the Caucasus, Website of Ministry of Foreign Affairs of Japan, 16.08.2021, https://www.mofa.go.jp/policy/oda/page_000010.html\#armenia
} 


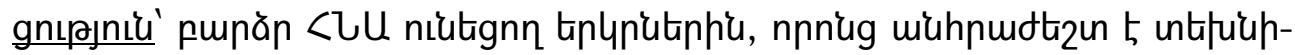

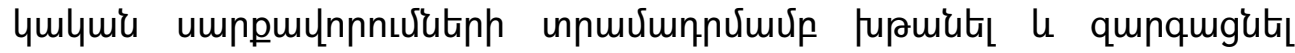

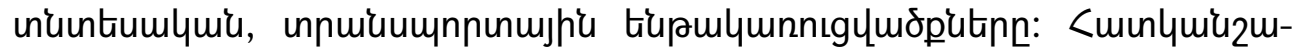

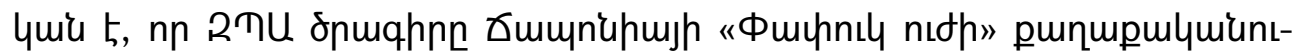

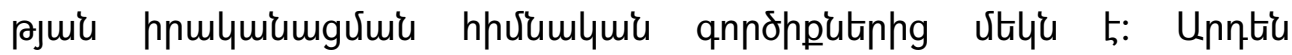

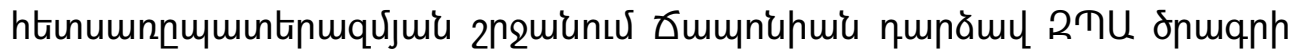
uर्utumúü nnünn:

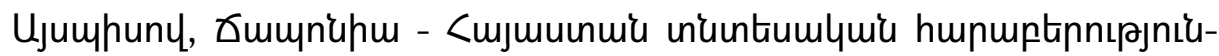

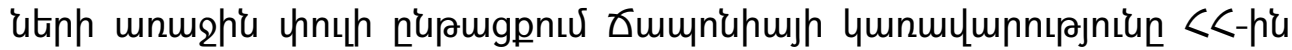

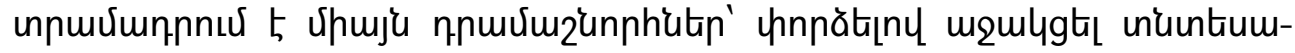

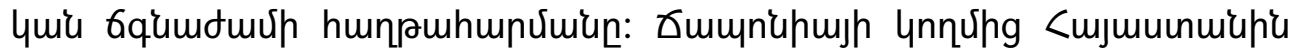

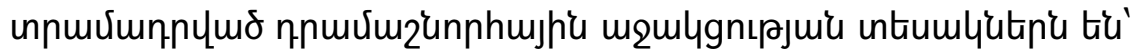

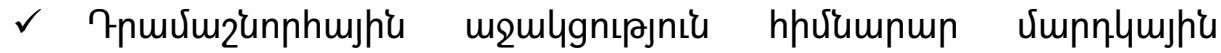
miulunuiuqujhu umpumqdtphu (Grassroots)",

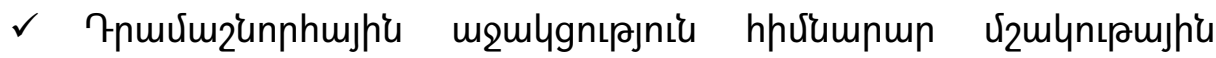
umpumqdtiphu (Grassroots),

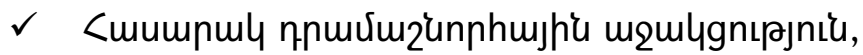

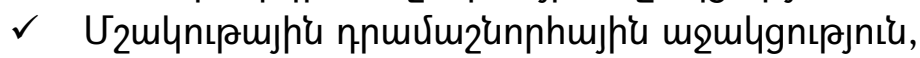

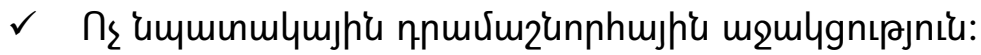

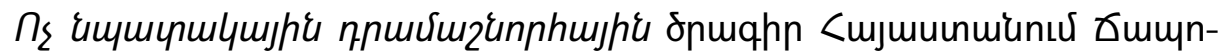

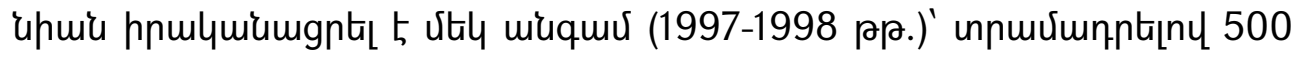

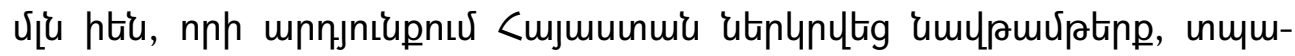

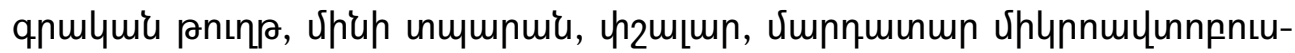

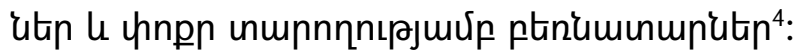

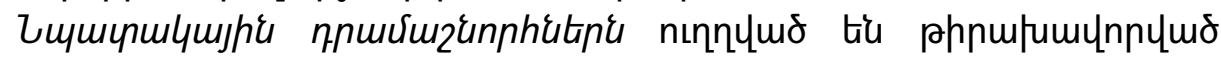

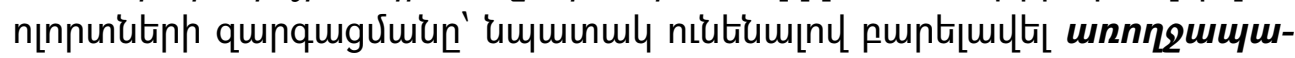

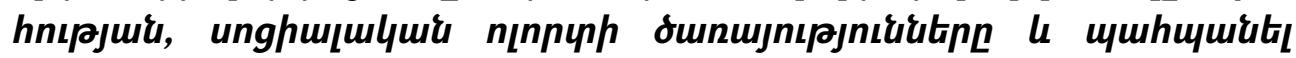
чишчи

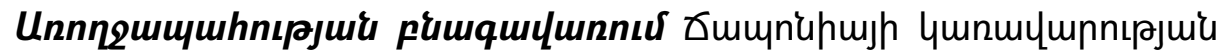

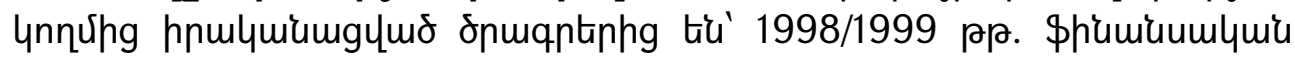

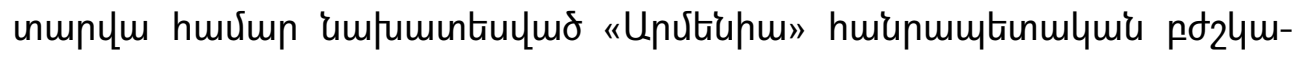

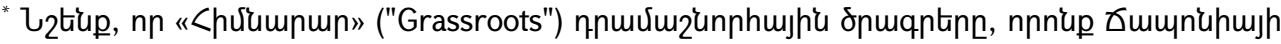

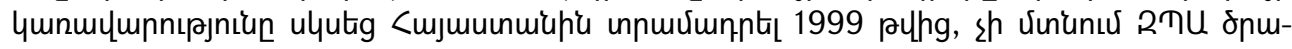

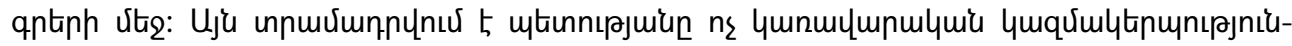
utiph (NGO) uhengnu:

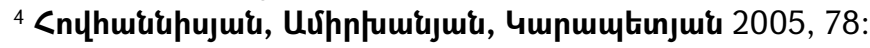




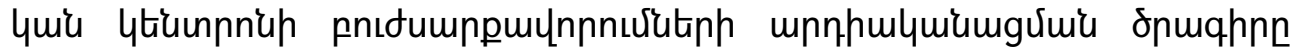

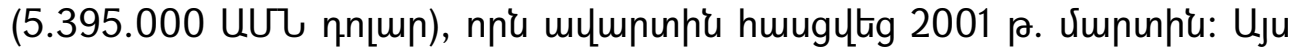

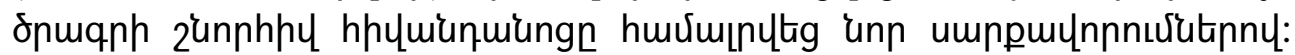

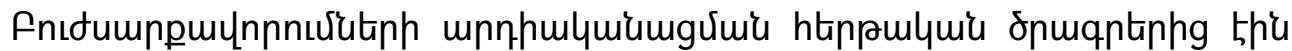

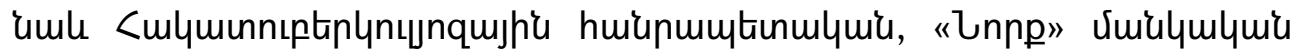

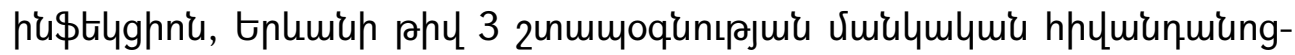

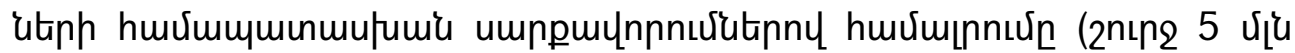
UUU пnцun) $)^{5}$ :

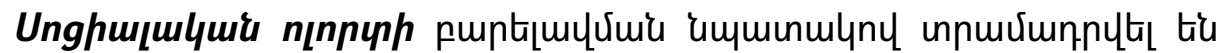

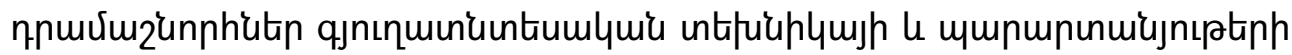

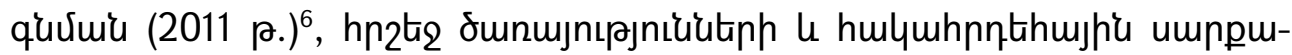

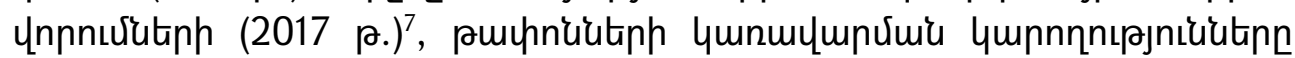

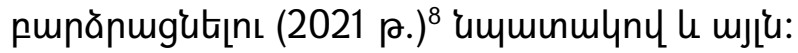

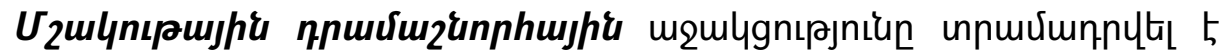

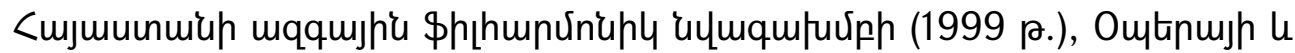

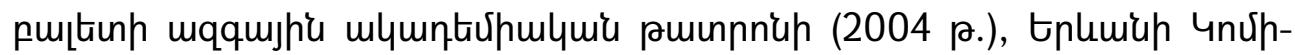

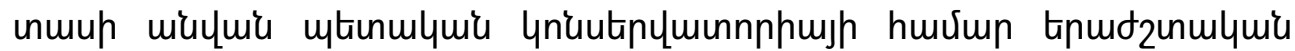

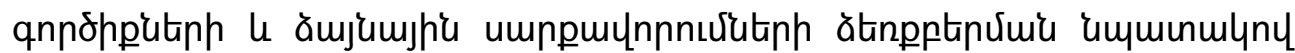

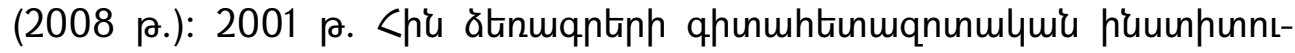

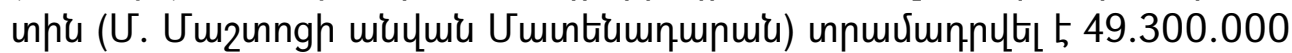

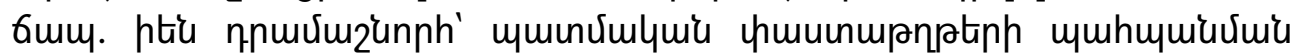

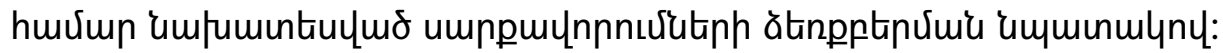

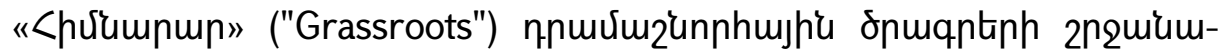

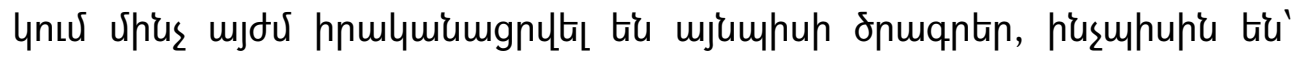

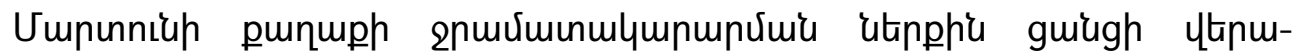

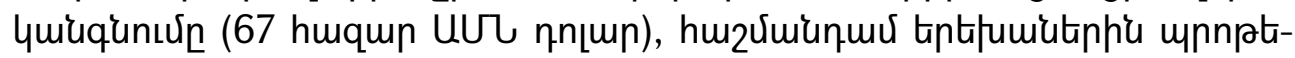

${ }^{5}$ Unıృ untønıu, 79:

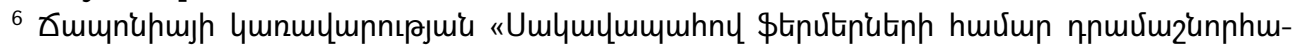

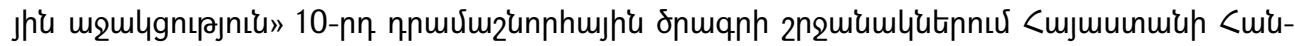

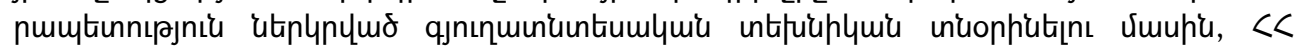

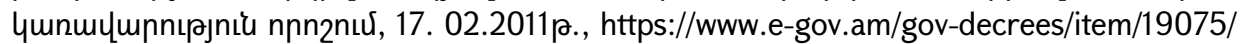

${ }^{7}$ Ex-Post Evaluation of Japanese ODA Grant Aid Project "The Project for Improvement of Fire Fighting Equipment in Yerevan City", Webiste of Japan International Cooperation Agency, https://www2.jica.go.jp/en/evaluation/pdf/2013_0867940_4_f.pdf

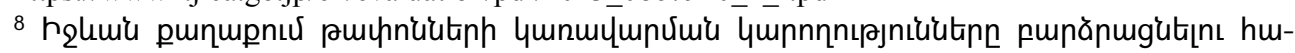

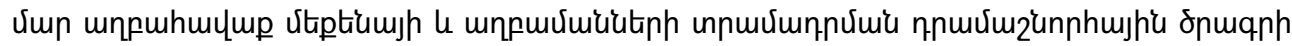

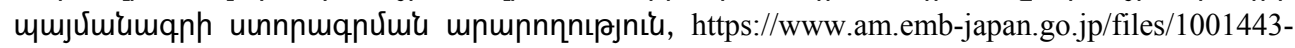
63.pdf?fbclid=IwAR1CP17DsZkXTeODfiDllC5iwHOONRoTEwueuFGi_V2WE7uR6Rq3nlK6U8U 


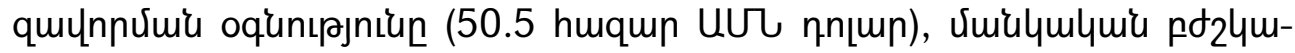

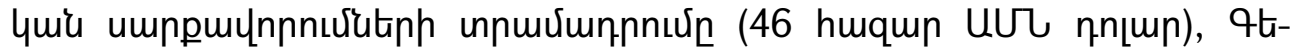

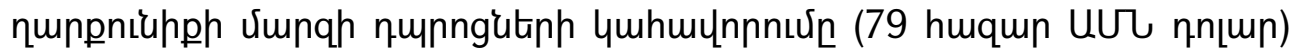

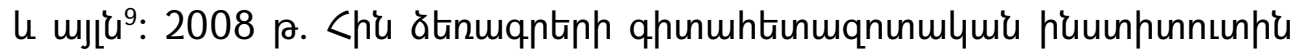

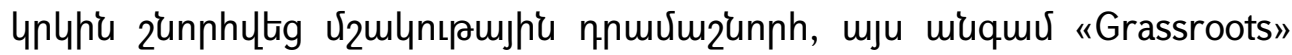

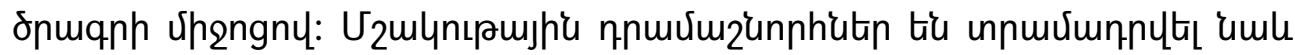

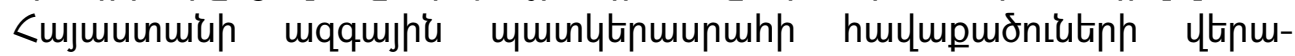

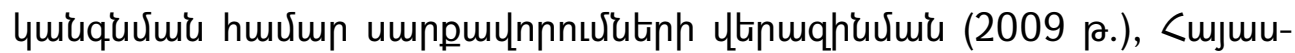

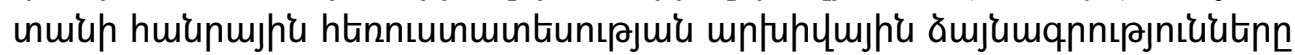

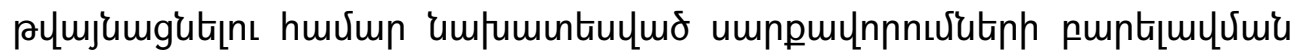

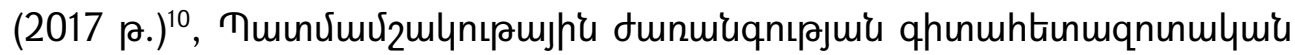

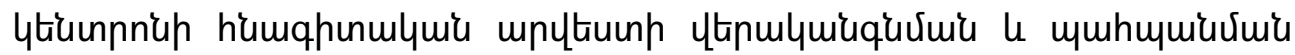

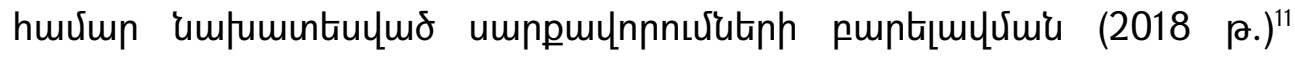
immunumin:

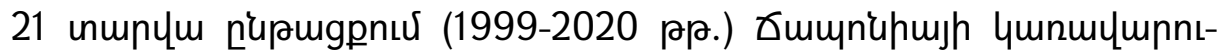

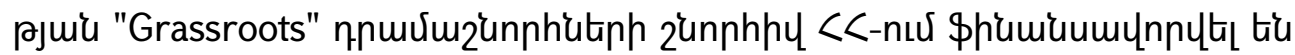

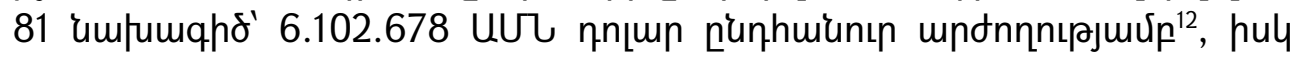

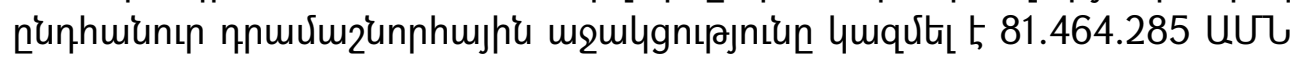
пnцun ${ }^{13}$ :

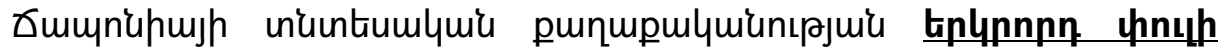

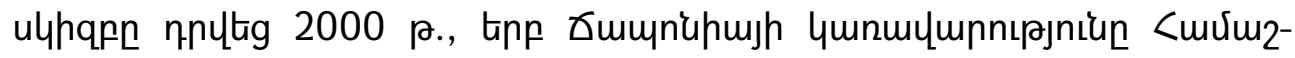

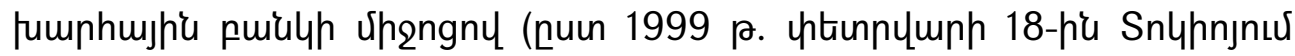

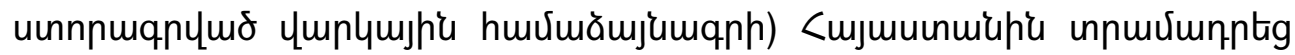

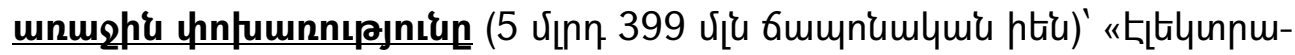

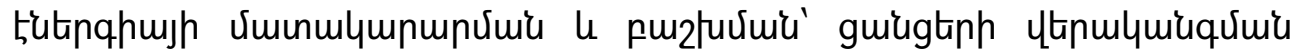

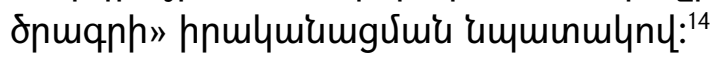

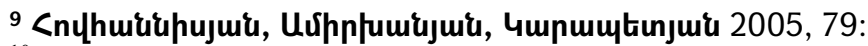

${ }^{10}$ Japan International Cooperation Agency (JICA) Signed on Grant Agreement with Armenia: The Archive of the Public Television will be Digitized, Webiste of Japan International Cooperation Agency, 30.03.2017, https://www.jica.go.jp/uzbekistan/english/office/topics/170330.html

${ }^{11}$ Grant Agreement Signed between JICA and the Government of the Republic of Armenia, Website of Japan International Cooperation Agency, 16.02.2018, https://www.jica.go.jp/uzbekistan/english/office/topics/press180216.html

12 Grant Assistance for Grassroots Human Security Projects (GGP), Website of Embassy of Japan in Armenia, 11.08.2021, https://www.am.emb-japan.go.jp/itpr_en/b_000195.html

13 Japan's Development Cooperation with the Republic of Armenia, Website of Ministry of Foreign Affairs of Japan, 2019, https://www.mofa.go.jp/files/000476406.pdf

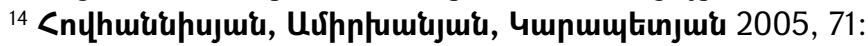




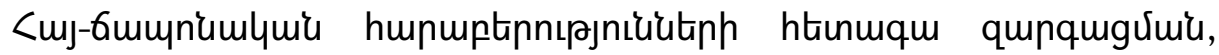

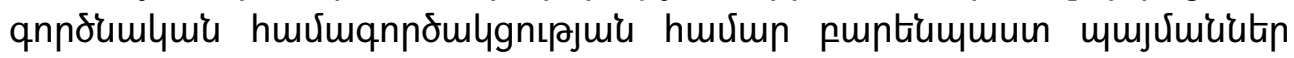

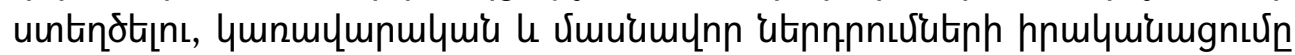

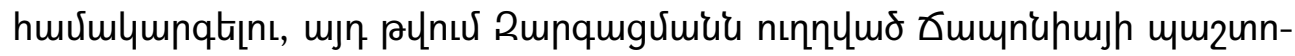

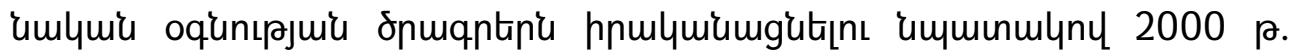

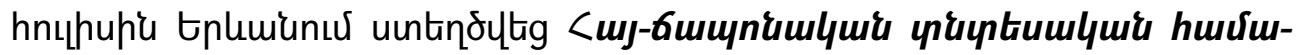

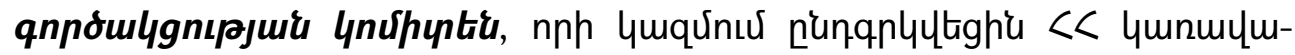

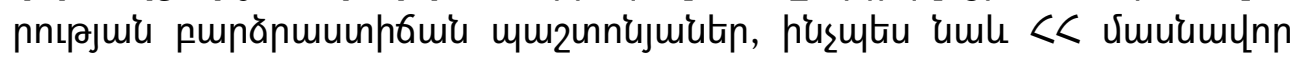

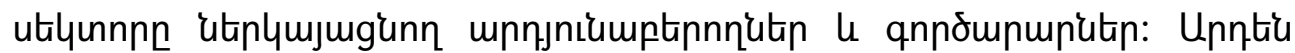

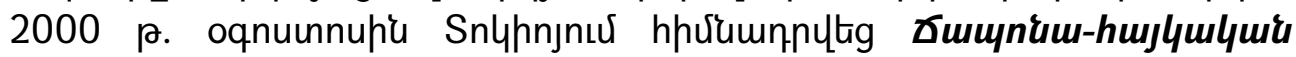

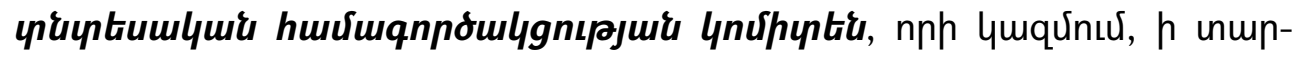

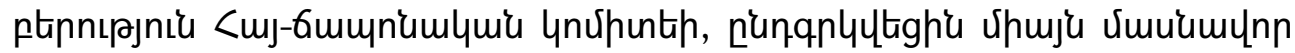

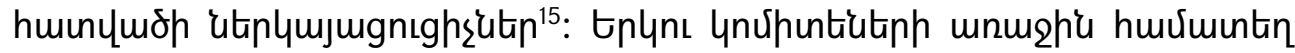

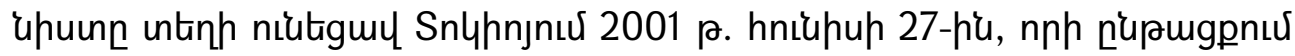

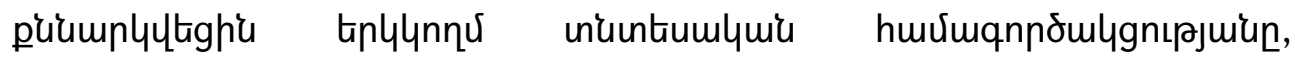

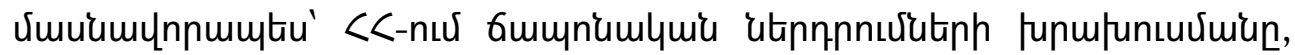

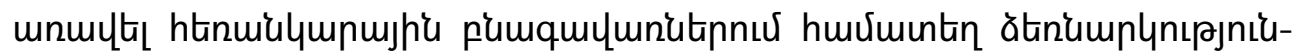

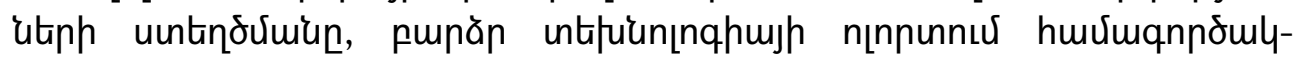

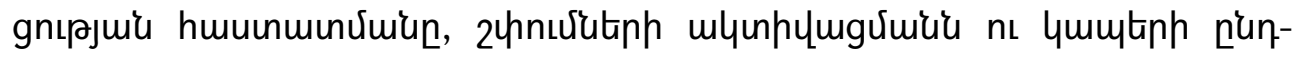

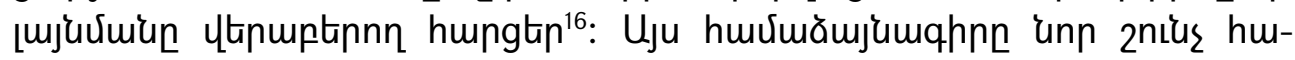

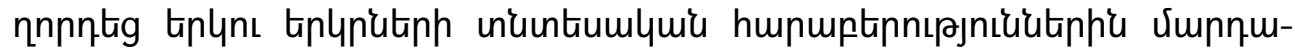

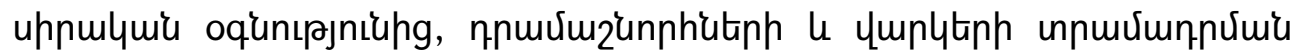

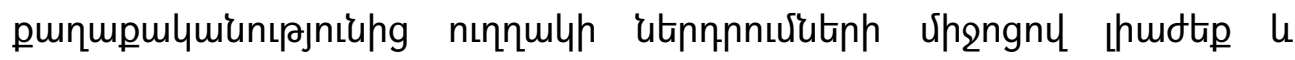

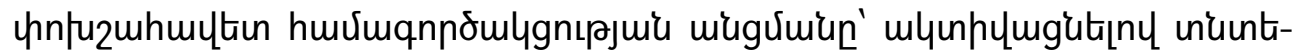

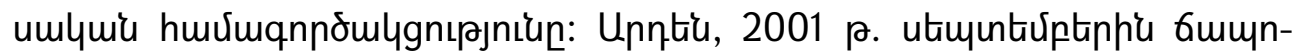

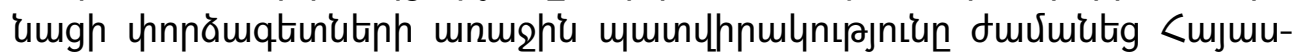

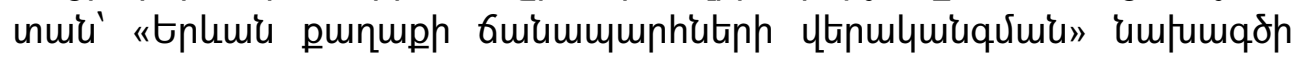

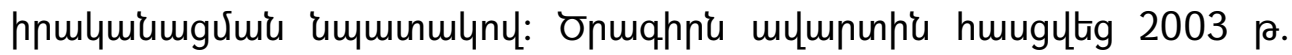
$\mathrm{u}_{2}$ 'uminu ${ }^{17}$ :

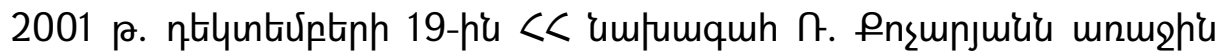

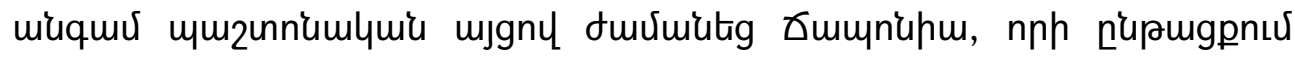

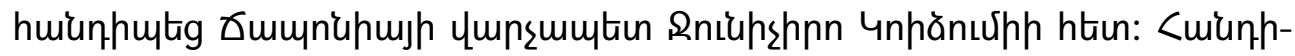

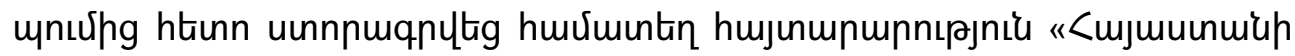

\footnotetext{
15 Unıృ untinnux, 71-72:

16 Nazarmuhamedov Bobur 2018: 177.

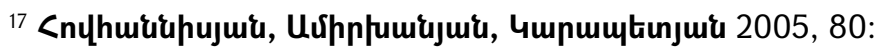




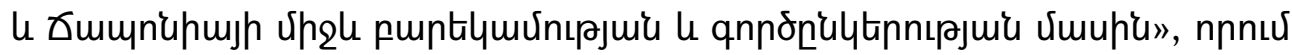

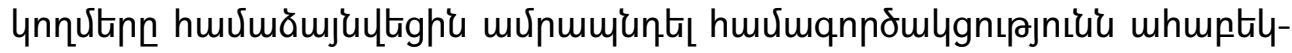

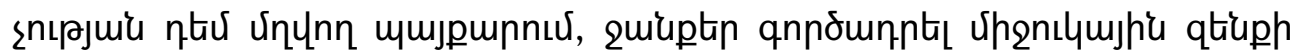

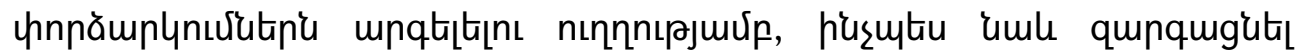

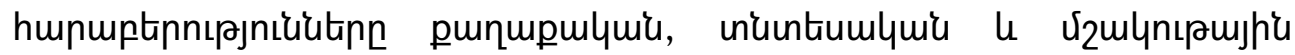
pumquulunutupnư 18 :

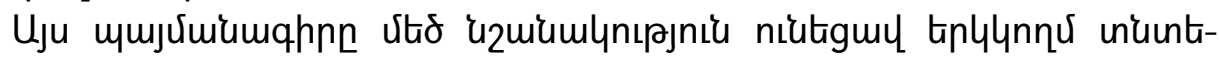

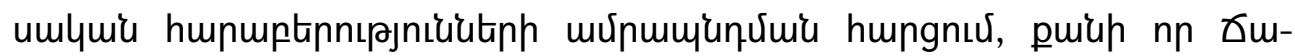

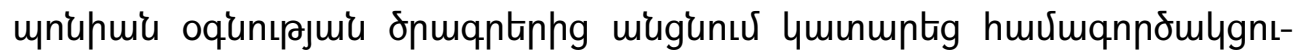

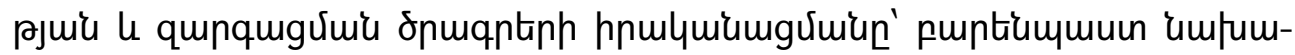

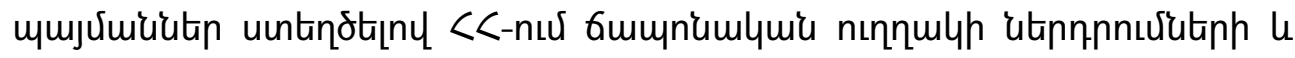

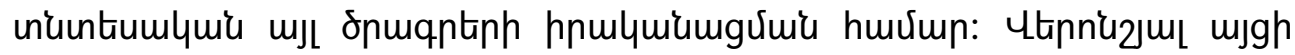

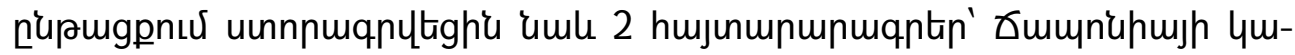

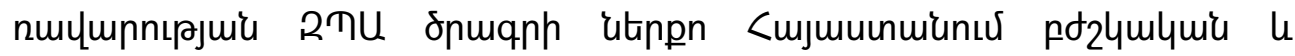

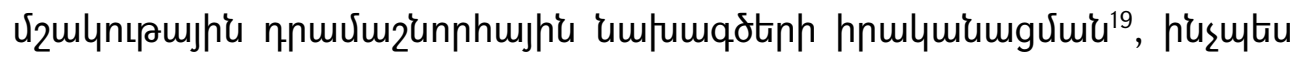

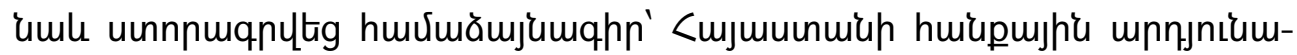

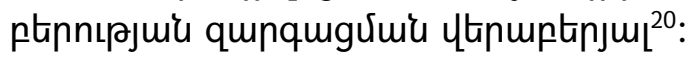

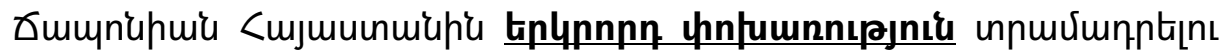

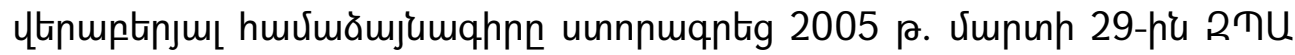

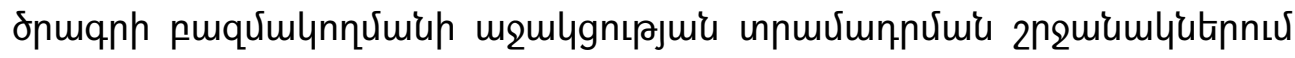

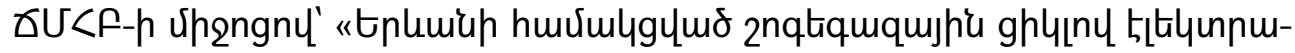

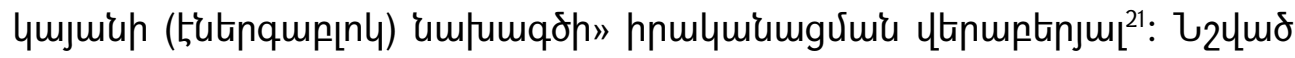

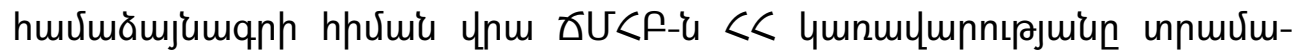

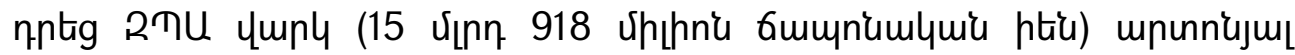

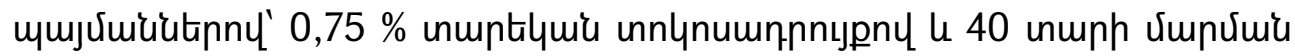

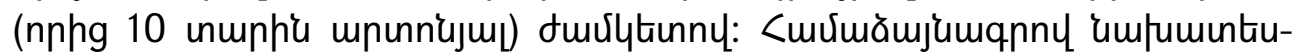

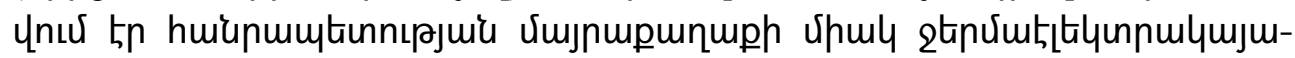

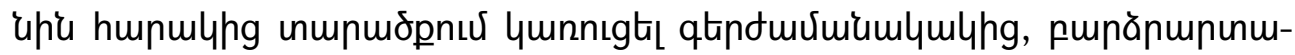

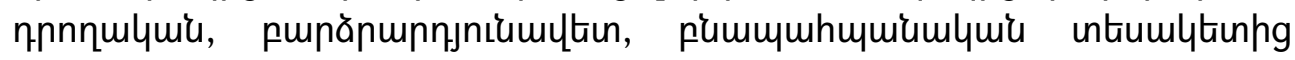

\footnotetext{
18 Joint Statement on Friendship and Partnership between Japan and the Republic of Armenia, Website of Ministry of Foreign Affairs of Japan, 19.12.2001, https://www.mofa.go.jp/region/europe/armenia/joint0111.html

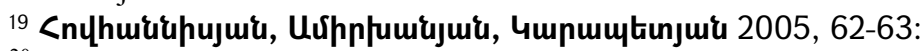

20 Joint Statement on Friendship and Partnership between Japan and the Republic of Armenia, Website of Ministry of Foreign Affairs of Japan, 19.12.2001, https://www.mofa.go.jp/region/europe/armenia/joint0111.html

${ }^{21}$ Activities in Armenia, JICA's Projects in Armenia, Website of Japan International Cooperation Agency, https://www.jica.go.jp/armenia/english/activities/index.html
} 


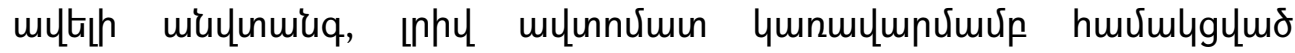

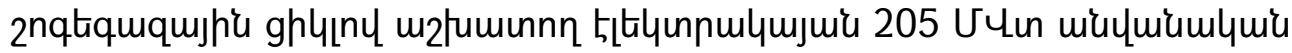

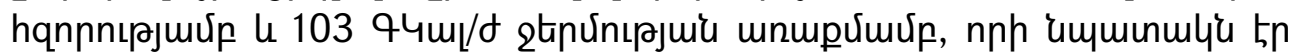

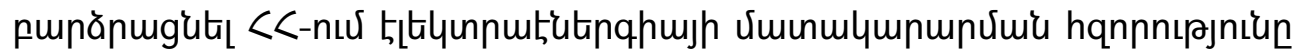

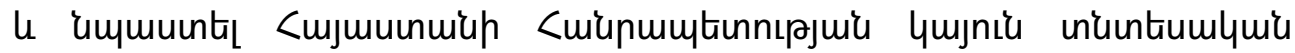
qunqugutuitu m6ht:

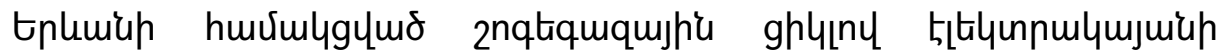

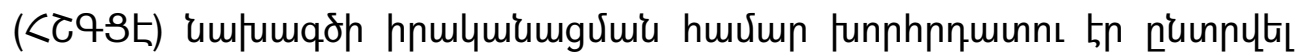
Gumnimlumí «Tokyo Electric Power Service Co., Ltd.» (TEPSCO) nulyt-

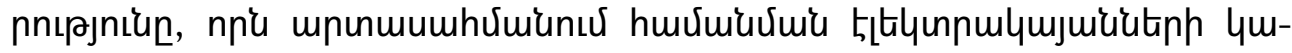

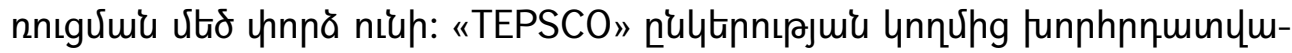

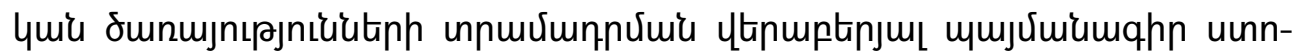

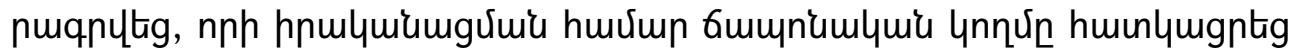

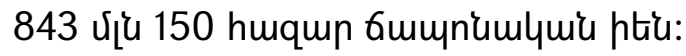

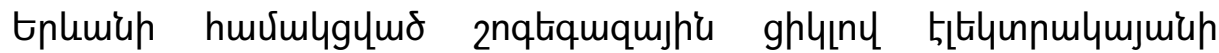

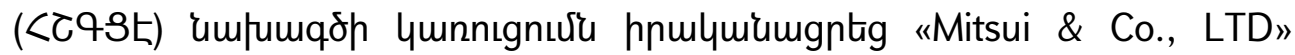
(бumnuhu) $\mathrm{l}$ "GS Engineering and Construction Corp.» (4nntiujh <uiu-

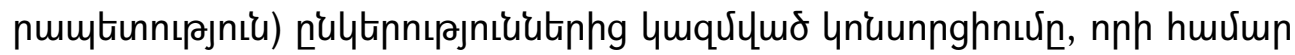

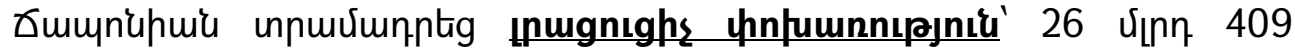

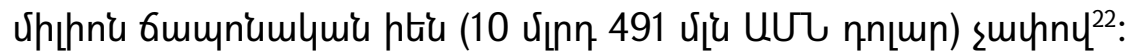

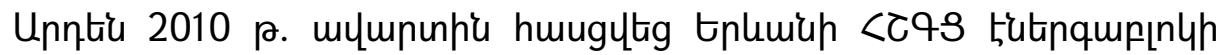

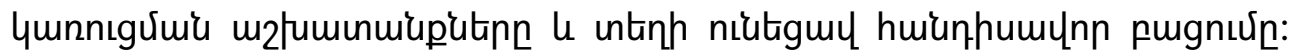

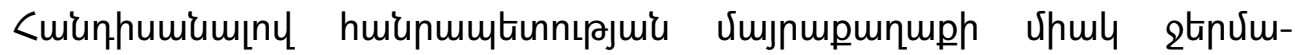

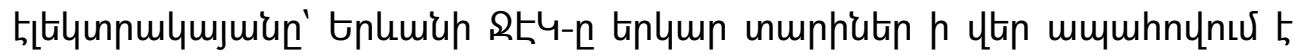

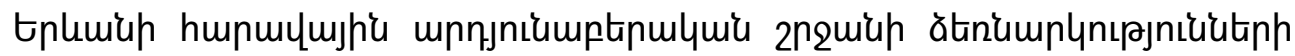

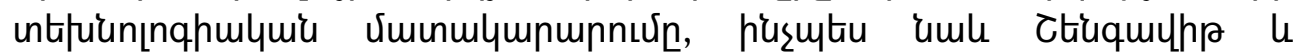

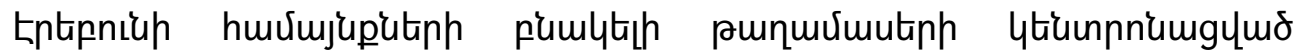

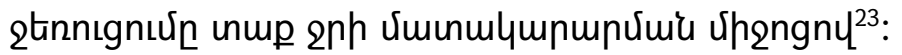

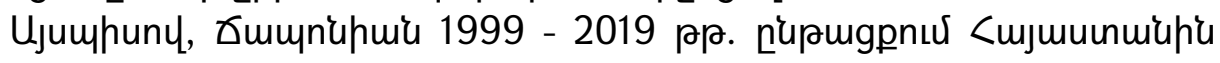

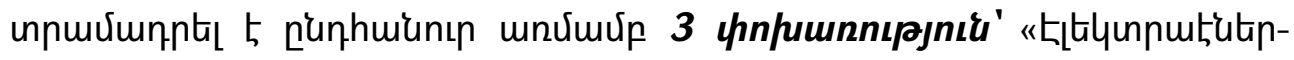

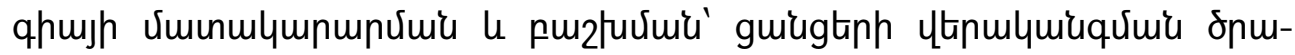

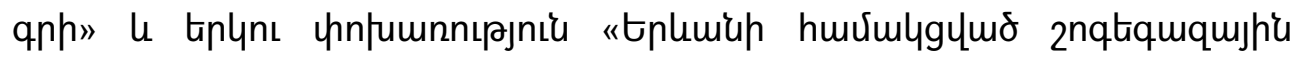

\footnotetext{
22 Unıju untinnux:

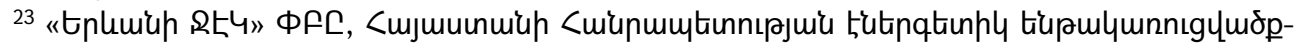

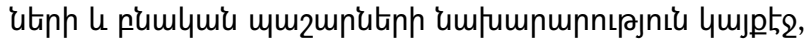

http://www.minenergy.am/page/531
} 


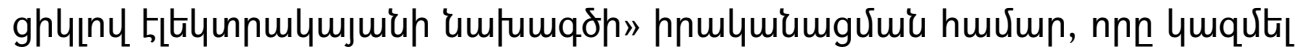

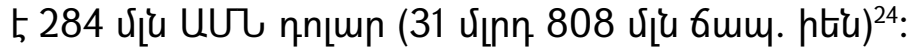

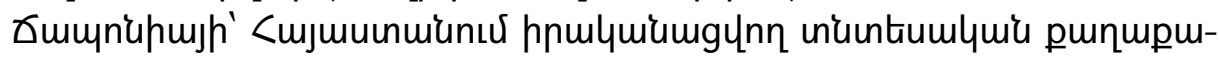

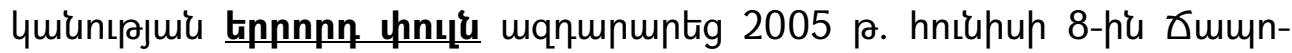

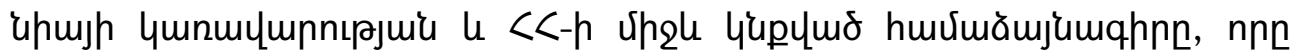

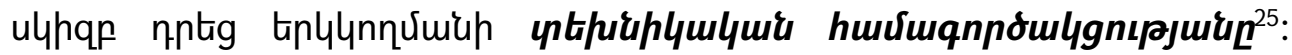

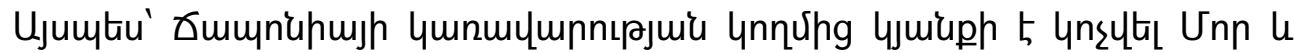

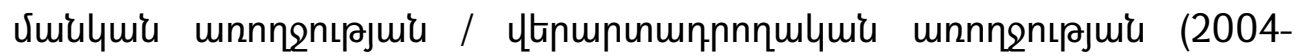

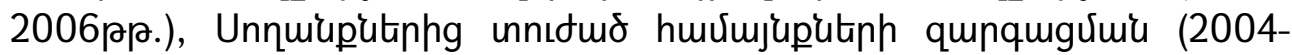

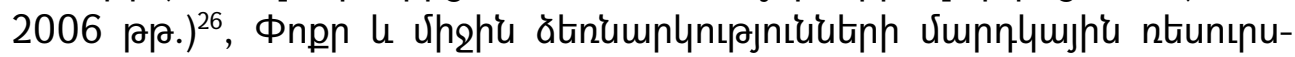

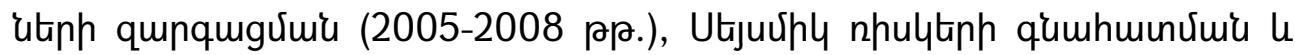

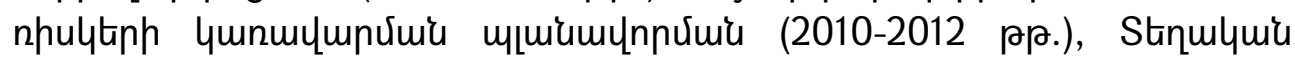

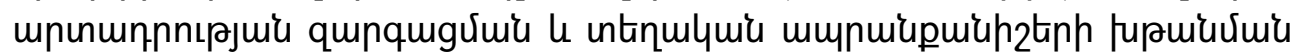

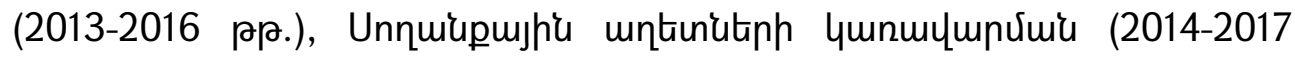

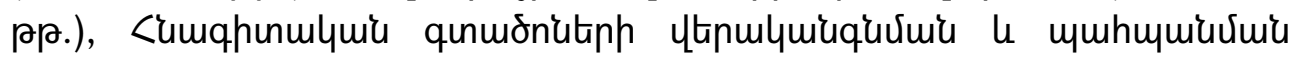

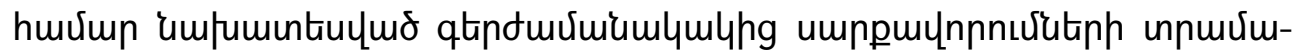

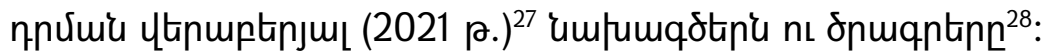

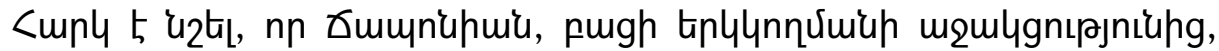

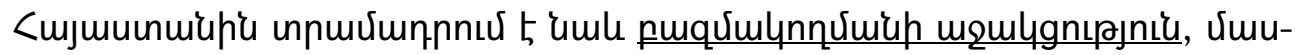

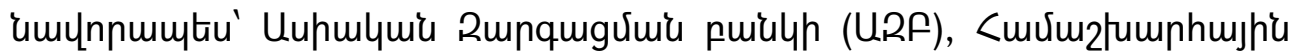

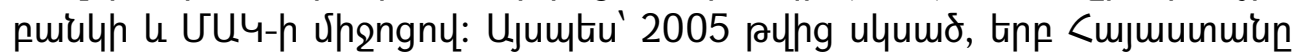

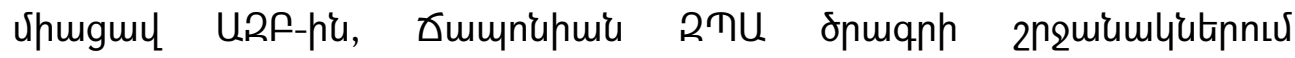

\footnotetext{
${ }^{24}$ Japan's Development Cooperation with the Republic of Armenia, Website of Ministry of Foreign Affairs of Japan, 2019, https://www.mofa.go.jp/files/000476406.pdf

${ }^{25}$ Agreement on Technical Cooperation between the Government of Japan and the Government of the Republic of Armenia, Website of Japan International Cooperation Agency, 08.06.2005, https://www.jica.go.jp/joureikun/act/actdata/110000118/current/FormEtc/13000018001000000002.pdf ${ }^{26}$ "<mjuuunwiuh <

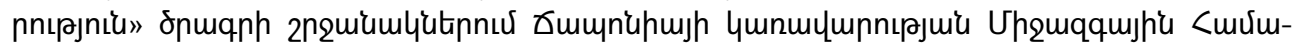

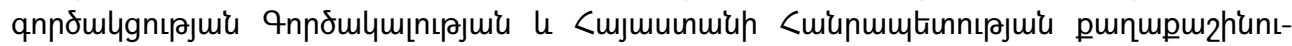

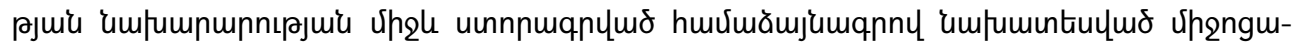

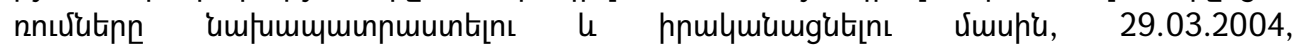
http://www.irtek.am/views/act.aspx?aid=24469

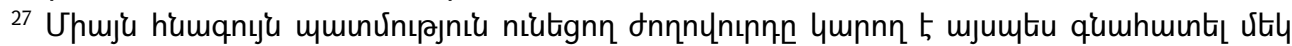

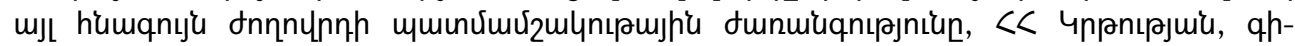

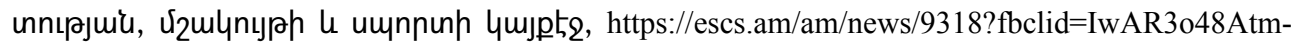
NrAKN-LQrxdgjc3oEiwG9jJRSfe5_GJ6quy3Kcir TCXjhA5vB8

${ }^{28}$ Activities in Armenia, JICA's Projects in Armenia, Website of Japan International Cooperation Agency, https://www.jica.go.jp/armenia/english/activities/index.html
} 


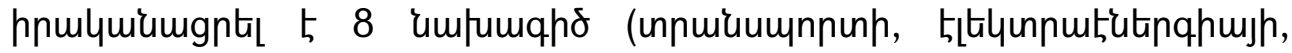

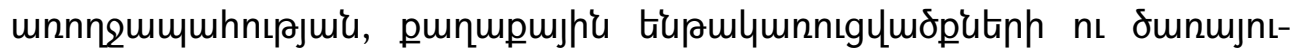

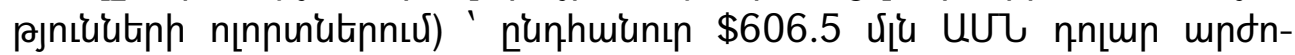

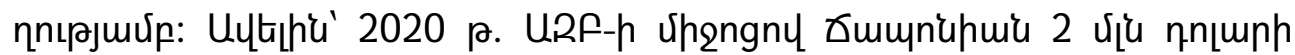

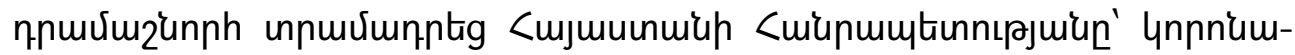

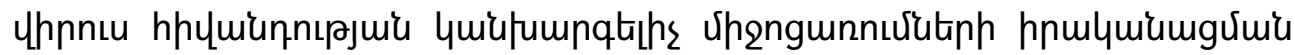

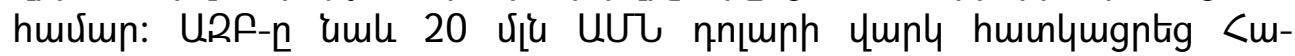

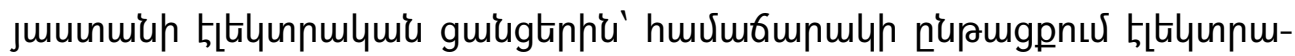

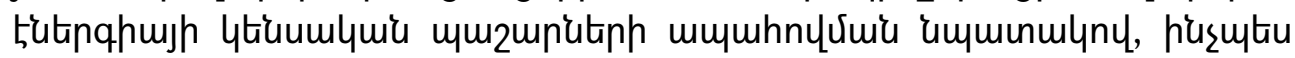

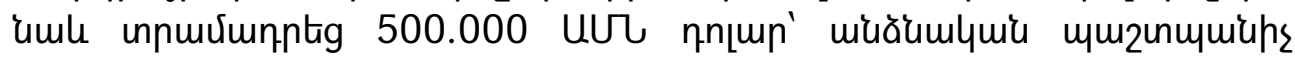

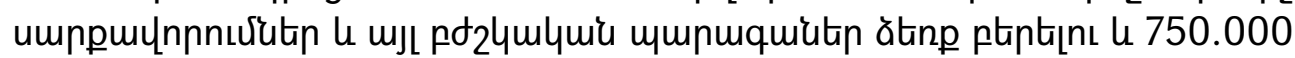

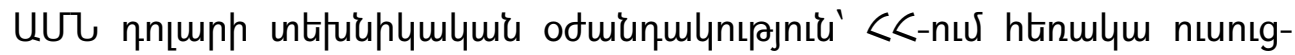

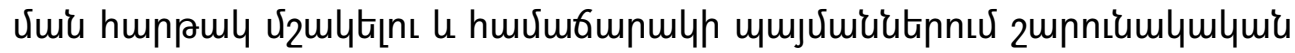

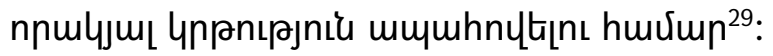

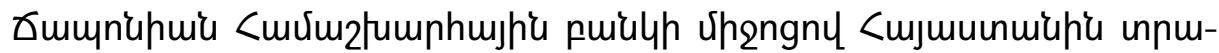

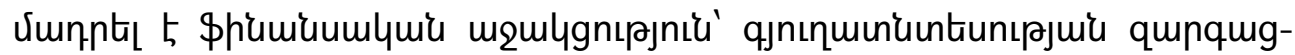

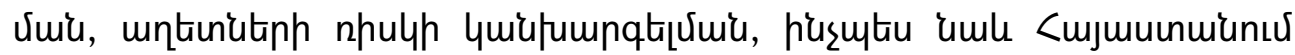

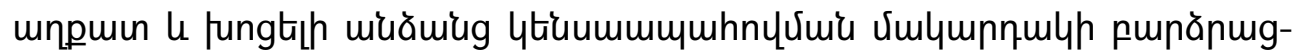

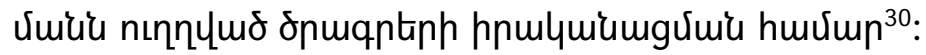

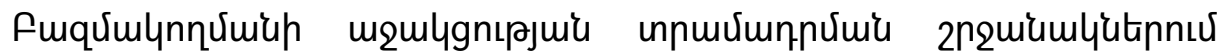

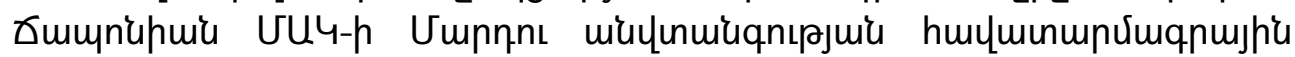

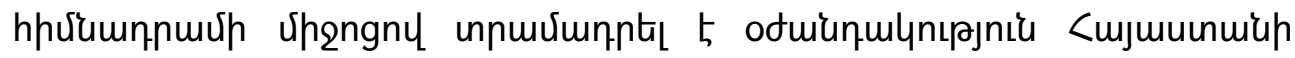

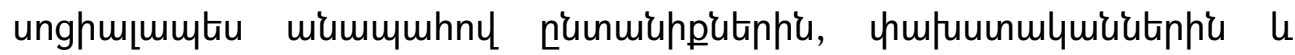

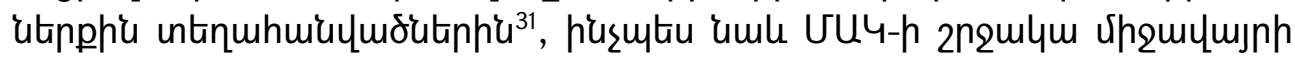

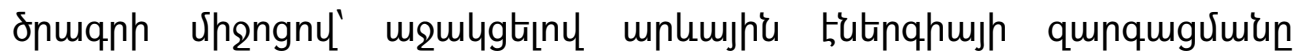

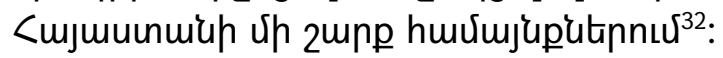

29 Armenia and ADB, Website of Asian Development Bank, https://www.adb.org/countries/armenia/overview

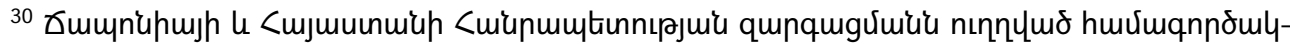

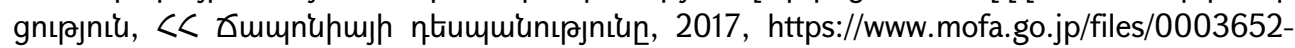
07.pdf

${ }^{31}$ Assistance by the United Nations Trust Fund for Human Security to the Project "Sustainable Livelihood for Socially Vulnerable Refugees, Internally Displaced and Local Families" in Armenia, Website of Ministry of Foreign Affairs of Japan, 02.03.2009, https://www.mofa.go.jp/announce/announce/2009/3/1188760_1130.html

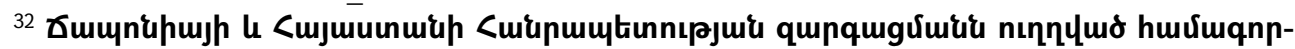

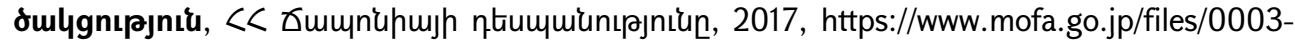
65207.pdf 


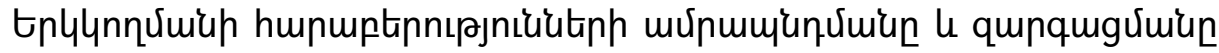

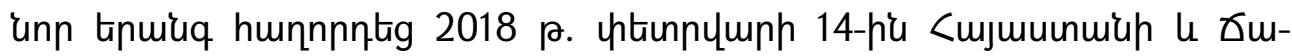

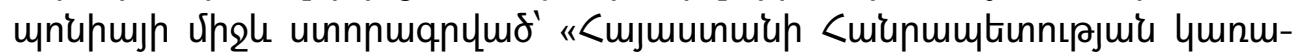

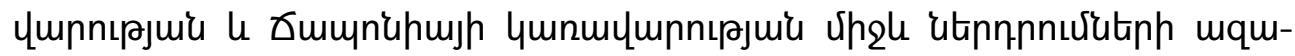

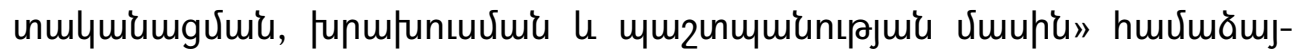

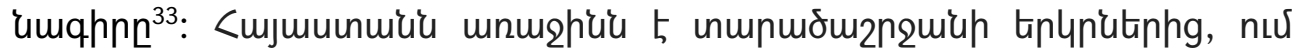

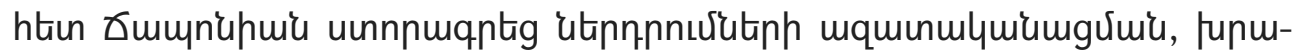

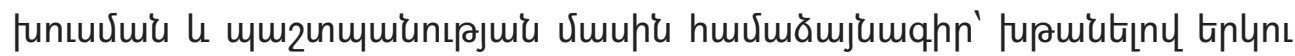

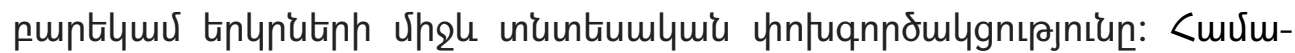

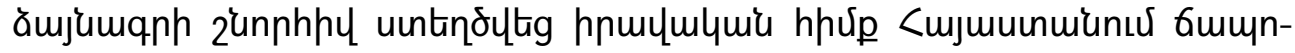

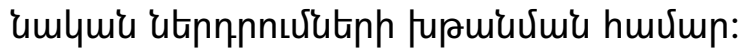

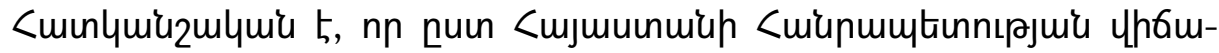

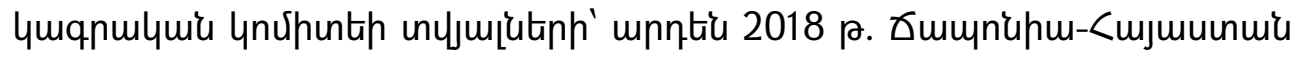

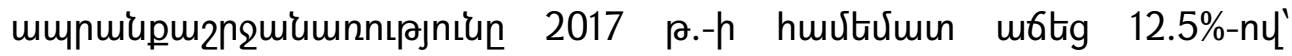

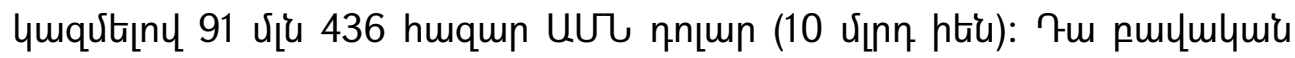

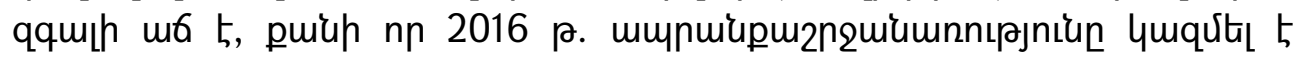

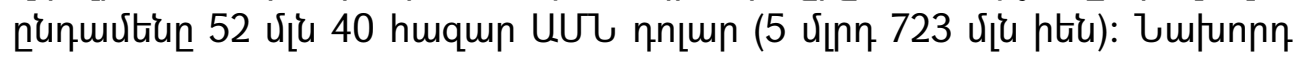

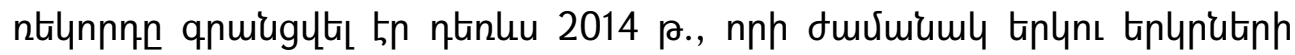

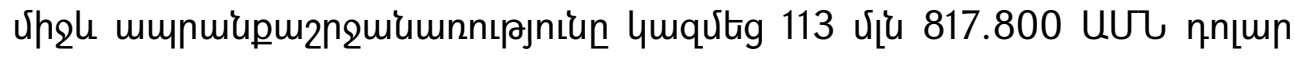
(12.5 únn htiu) (unti'u Unjnıuml 2) $)^{34}$ :

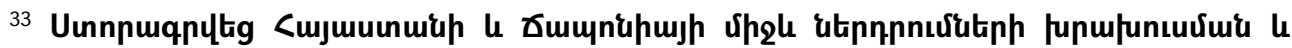

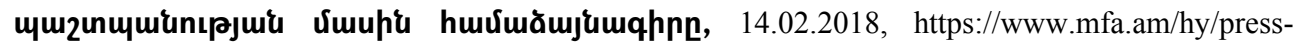
releases/2018/02/14/fm-japan-agreement/7946

34 Japan Ambassador: Armenia has Truly Unique Position in the World Trade System, 23.02.2019, https://news.am/eng/news/497328.html 


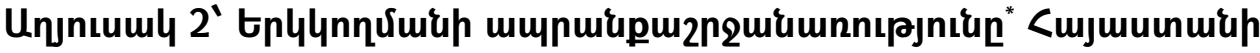

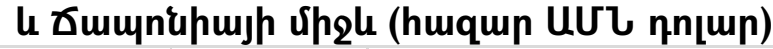

\begin{tabular}{|c|c|c|c|c|}
\hline \multicolumn{2}{|c|}{ Ununuhuutunuর } & Utnlynnux & 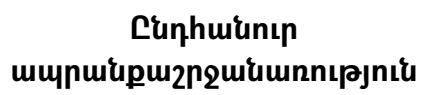 & \\
\hline 2014 & & 49.0 & 113668.8 & 113817 \\
\hline 2015 & & 26.9 & 57605.8 & 61133 \\
\hline 2016 & & 79.3 & 51661.4 & 52040 \\
\hline 2017 & & 66.1 & 81.021 .2 & 81387.3 \\
\hline 2018 & & 75.4 & 99060.6 & 99436 \\
\hline
\end{tabular}

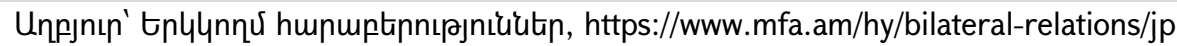

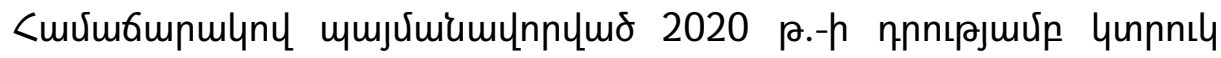

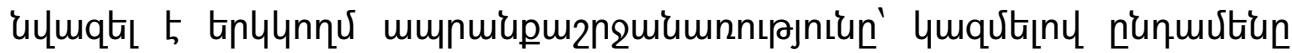

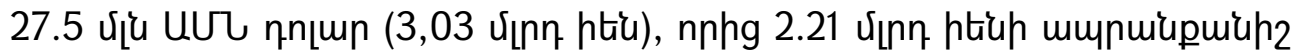

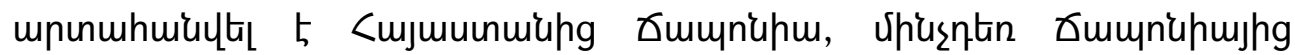

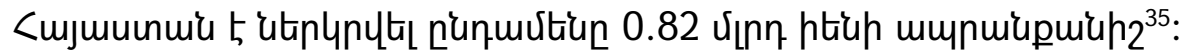

\section{Uúuhnцnus}

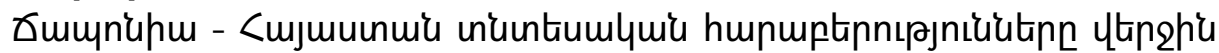

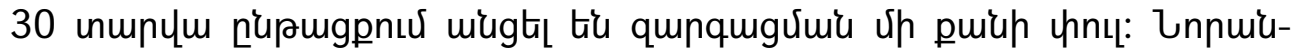

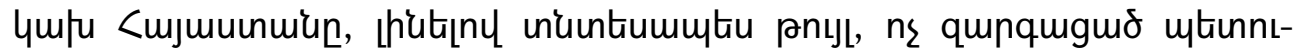

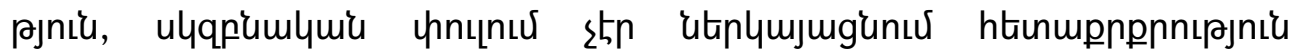

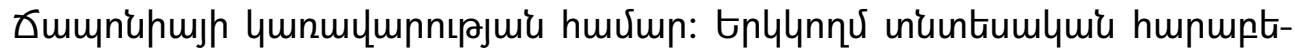

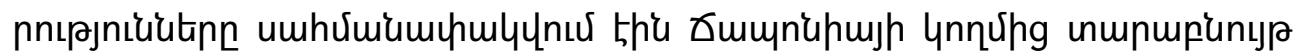

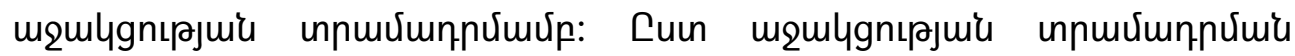

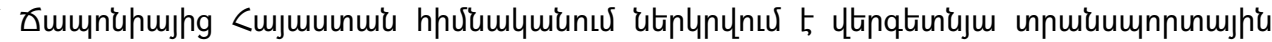

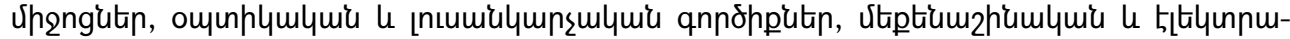

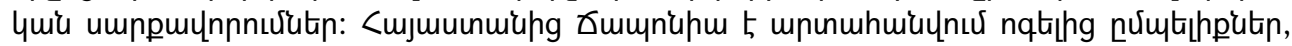

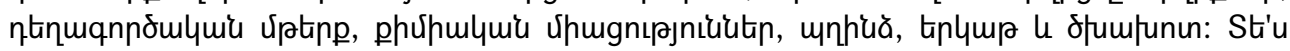
Bilateral Relations, Japan, https://www.mfa.am/en/bilateral-relations/jp

35 Japan-Armenia Relations (Basic Data), Website of Ministry of Foreign Affairs of Japan, https://www.mofa.go.jp/region/europe/armenia/data.html\#section1
} 


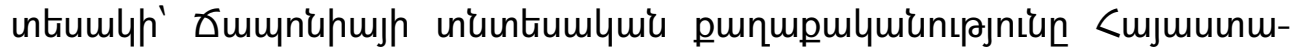

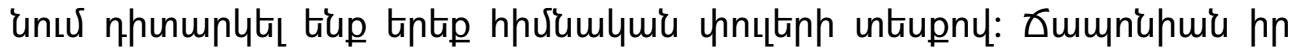

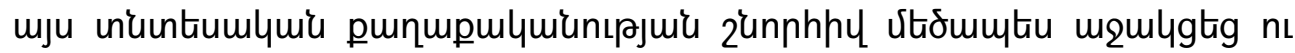

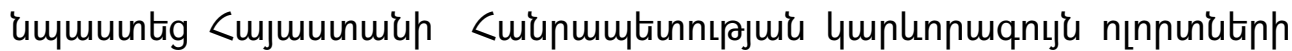

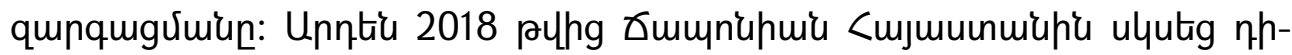

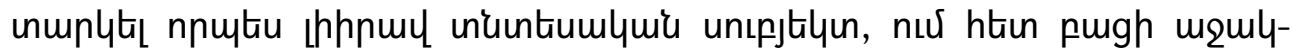

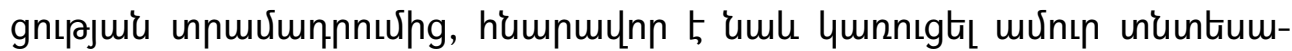

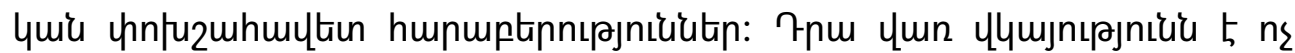

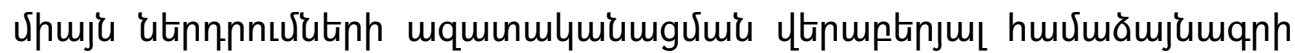

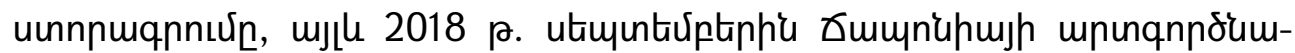

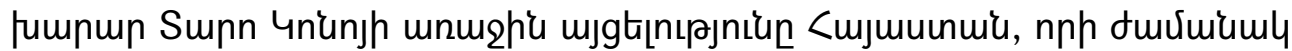

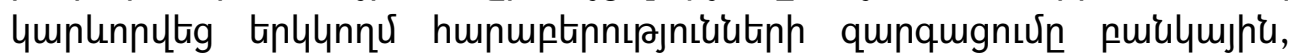

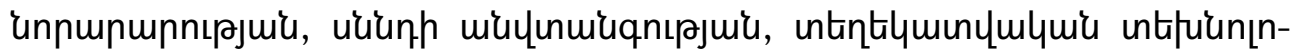

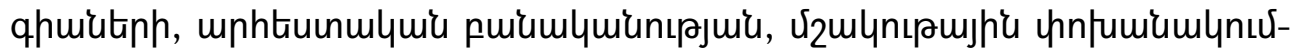

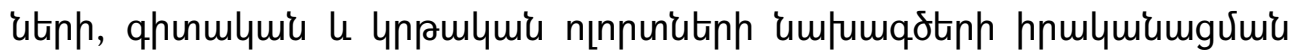

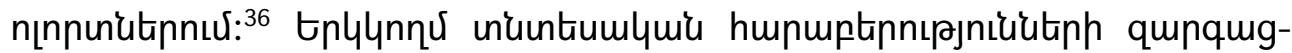

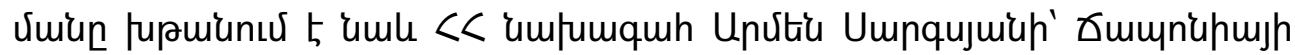

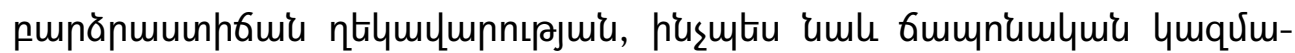

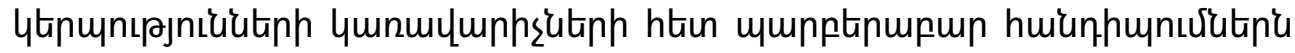

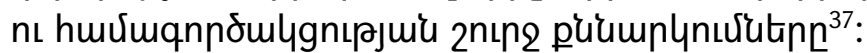

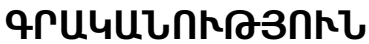

"tphumah RE4»

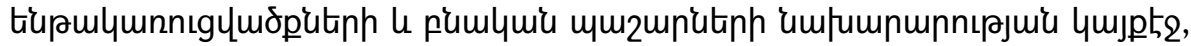

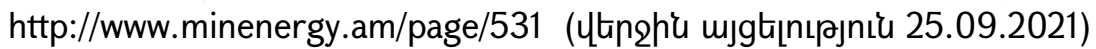

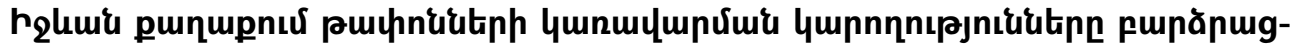

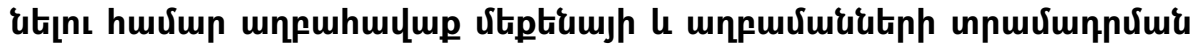

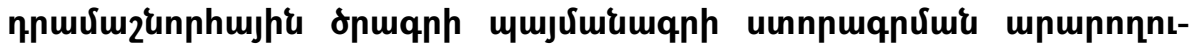
pjnıl, 2020, https://www.am.emb-japan.go.jp/files/100144363.pdf?fbclid=IwAR1CP17DsZkXTeODfiDIIC5iwHOONRoTEwueuFGi_V2WE7uR6Rq3nIK6U8U

\footnotetext{
${ }^{36}$ President Sarkissian hosted the Minister of Foreign Affairs of Japan, Website of the President of the Republic of Armenia, 03.09.2018, https://www.president.am/en/pressrelease/item/2018/09/03/President-Armen-Sarkissian-met-with-minister-of-foreign-affairs-of-JapanTaro-Kono/

${ }^{37}$ Armenia wants to impart new quality to the relations with Japan and start a new page of cooperation President Sarkissian met with the Prime Minister of Japan Suga Yoshihide, Website of the President of the Republic of Armenia, 24.07.2021, https://www.president.am/en/pressrelease/item/2021/07/24/President-Armen-Sarkissian-met-with-Yoshihide-Suga/
} 


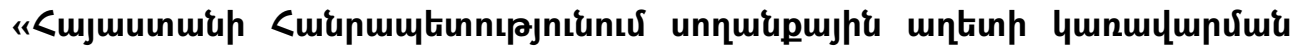

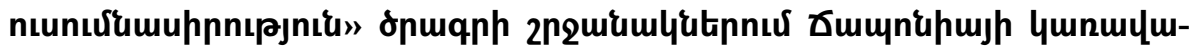

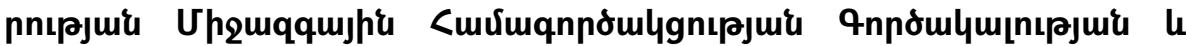

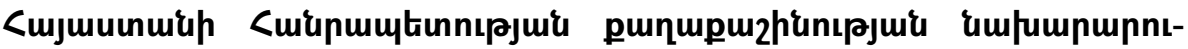

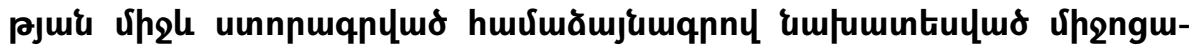

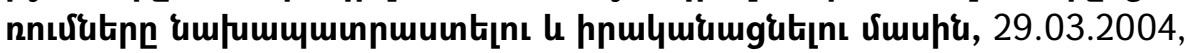

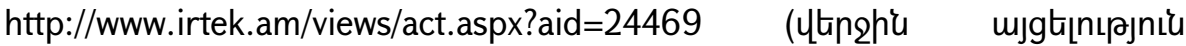
25.09.2021)

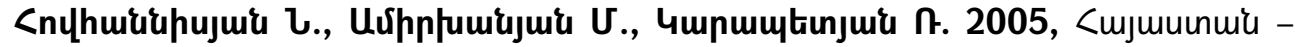

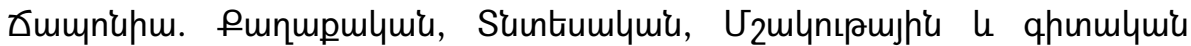

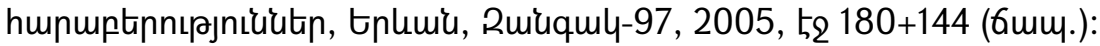

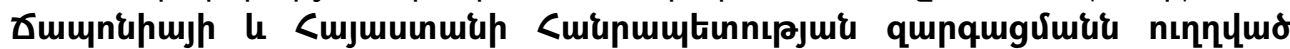
huưumqnndulgnıpjniu, Website of Ministry of Foreign Affairs of Japan, 2017, https://www.mofa.go.jp/files/000365207.pdf

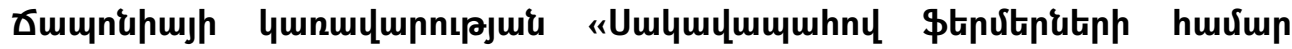

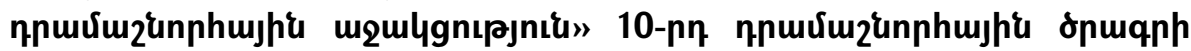

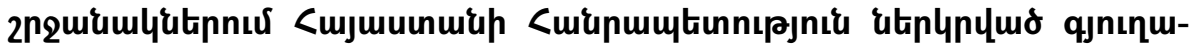

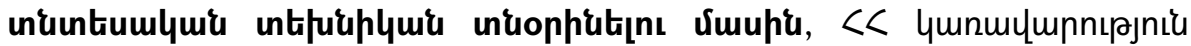
nnn2nuर, 17.02.2011p., https://www.e-gov.am/gov-decrees/item/19075/

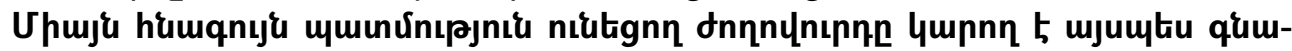

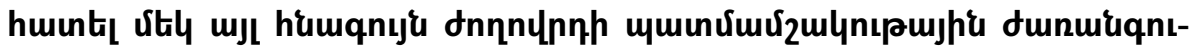

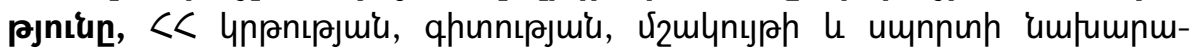
nnıرuiu 4mjpt2, https://escs.am/am/news/9318?fbclid=IwAR3o48AtmNrAKNLQrxdgjc3oEiwG9jJRSfe5_GJ6quy3KCirTCXjhA5vB8 (цtinghu ujgtınıpjnıu 05.10.2021)

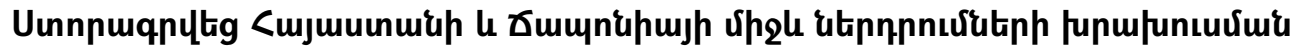

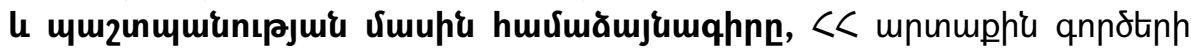

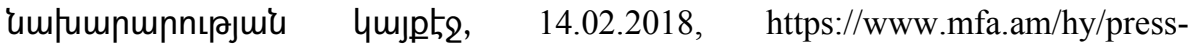

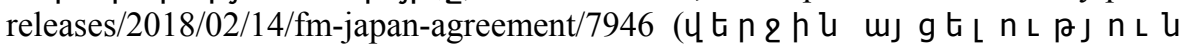
25.09.2021)

Activities in Armenia, JICA's Projects in Armenia, Website of Japan International Cooperation Agency, https://www.jica.go.jp/armenia/english/activities/index.html (last access on 25.09.2021)

Agreement on Technical Cooperation between the Government of Japan and the Government of the Republic of Armenia, Website of Japan International Cooperation

Agency, https://www.jica.go.jp/joureikun/act/actdata/110000118/current/FormEtc/130000180 01000000002.pdf (last access on 25.09.2021)

Armenia and ADB, Website of Asian Development Bank, https://www.adb.org/countries/armenia/overview (last access on 25.09.2021)

Armenia Wants to Impart New Quality to the Relations with Japan and Start a New Page of Cooperation President Sarkissian Met with the Prime Minister of Japan Suga Yoshihide, Website of the President of the Republic of Armenia, 
24.07.2021, https://www.president.am/en/press-release/item/2021/07/24/PresidentArmen-Sarkissian-met-with-Yoshihide-Suga/

Assistance by the United Nations Trust Fund for Human Security to the Project "Sustainable Livelihood for Socially Vulnerable Refugees, Internally Displaced and Local Families" in Armenia, Website of Ministry of Foreign Affairs of Japan, 02.03.2009,

https://www.mofa.go.jp/announce/announce/2009/3/1188760_1130.html (last access on 25.09.2021)

Bilateral Relations, Japan, Website of Foreign Affairs of the Republic of Armenia, https://www.mfa.am/en/bilateral-relations/jp (last access on 25.09.2021)

Ex-Post Evaluation of Japanese ODA Grant Aid Project "The Project for Improvement of Fire Fighting Equipment in Yerevan City", Website of Japan International Cooperation Agency, https:/www2.jica.go.jp/en/evaluation/pdf/2013_0867940_4_f.pdf (last access on 05.10.2021)

Grant Agreement Signed between JICA and the Government of the Republic of Armenia, Website of Japan International Cooperation Agency, 16.02.2018, https://www.jica.go.jp/uzbekistan/english/office/topics/press180216.html

Grant Assistance for Grassroots Human Security Projects (GGP), Website of Embassy of Japan in Armenia, 11.08.2021, https://www.am.emb-japan.go.jp/itpr_en/b_000195.html (last access on 05.10.2021)

Japan Ambassador: Armenia has Truly Unique Position in the World Trade System, Armenian News-NEWS.am Information-analytical Agency, 23.02.2019, https://news.am/eng/news/497328.html (last access on 05.10.2021)

Japan International Cooperation Agency (JICA) Signed on Grant Agreement with Armenia: The Archive of the Public Television will be Digitized, Website of Japan International Cooperation Agency, https://www.jica.go.jp/uzbekistan/english/ office/topics/170330.html (last access on 05.10.2021)

Japan's Development Cooperation with the Republic of Armenia, Website of Ministry of Foreign Affairs of Japan, 2019, https://www.mofa.go.jp/files/000476406.pdf (last access on 25.09.2021)

Japan-Armenia Relations (Basic Data), Website of Ministry of Foreign Affairs of Japan, https://www.mofa.go.jp/region/europe/armenia/data.html\#section1 (last access on 25.09.2021)

Japan's ODA-Fifty Years in the Front Lines, Website of Japan International Cooperation Agency,https://www.jica.go.jp/english/publications/reports/network/archive_2004/v ol_25_2.html (last access on 25.09.2021)

Joint Statement on Friendship and Partnership between Japan and the Republic of Armenia, Website of Ministry of Foreign Affairs of Japan, 19.12.2001, https://www.mofa.go.jp/region/europe/armenia/joint0111.html (last access on 25.09.2021)

Nazarmuhamedov B. 2018, Japan's ODA Policy toward Central Asia and the Caucasus an Analysis of Japanese Assistance to Economic Development in Kyrgyzstan and Armenia, "Journal of International and Advanced Japanese Studies, University of Tsukuba", Volume 10 ONLINE/March 2018, p. 169-185, http://japan.tsukuba.ac.jp/research/JIAJS10_ONLINE03_Nazarmuhamedov.pdf (last access on 05.10.2021)

ODA by Region Central Asia and the Caucasus, Website of Ministry of Foreign Affairs of Japan, 16.08.2021, 
https://www.mofa.go.jp/policy/oda/page_000010.html\#armenia (last access on 25.09.2021).

President Sarkissian Hosted the Minister of Foreign Affairs of Japan, Website of the President of the Republic of Armenia, 03.09.2018, https://www.president.am/en/press-release/item/2018/09/03/President-Armen-Sarkissian-met-with-minister-offoreign-affairs-of-Japan-Taro-Kono/

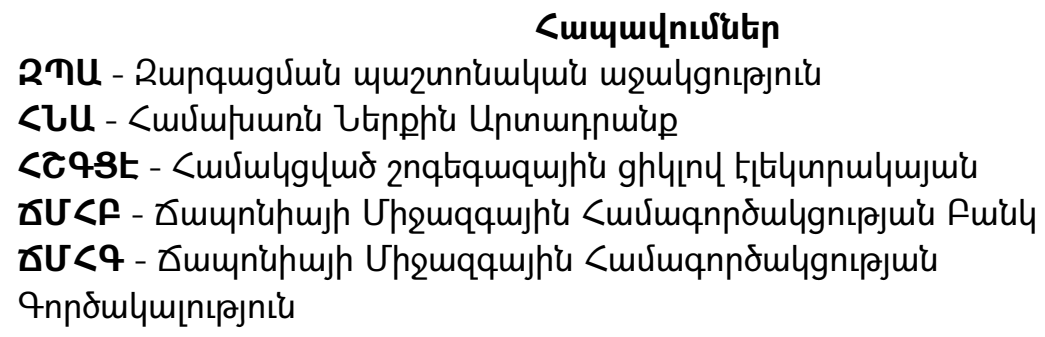

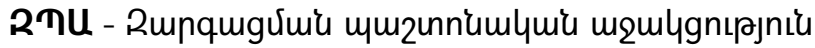

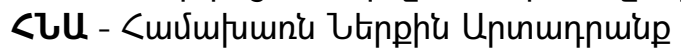

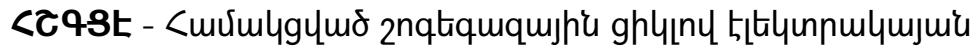

వU

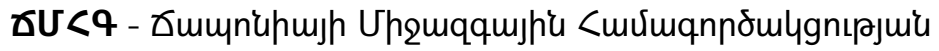

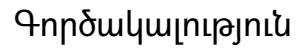

\author{
buu <unnıpjniquui

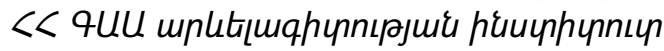 \\ yeva.harutyunyan85@gmail.com
}

\title{
JAPAN'S ECONOMIC POLICY IN ARMENIA (1992-2021)
}

\author{
Yeva Harutyunyan
}

Keywords: Japan, Armenia, Economic relations, Official Development Assistance, grants, loan, technical assistance.

The purpose of this article is to show the economic policy of Japan towards Armenia after the establishment of Armenian-Japanese diplomatic relations. The article presents three phases of Japan's economic policy, taking into account the type of assistance provided by Japan to Armenia, which is correlated with Armenia's economic and GDP's growth.

The first phase covers 1992-99, during which bilateral economic relations were limited to grant assistance provided by Japan to Armenia, with the aim of supporting the economic recovery of the newly independent state and ensuring sustainable economic growth.

The second phase (2000-04) marked the beginning of economic cooperation between the two countries. Due to its stable economic growth, Armenia received loans from Japan for developing economic infrastructures.

During the third phase (2005- up to date) Japan has expanded its cooperation with the Republic of Armenia and begun to provide technical equipment for the development of targeted sectors. At this stage, Japan clearly defined the direction of its assistance and the sectors necessary for the stimulation of the Armenian economy, in particular, the energy sector, small and medium-sized businesses, as well as the development of villages. Japan also greatly supports Armenia by providing Japanese technologies for risk prevention in natural disaster zones and organizing training courses and discussion- meetings for 
Armenian specialists aimed to promote capacity building on the implementation of disaster risk reduction and emergency situations.

Since 2018, Japan has begun to consider Armenia as a full-fledged economic entity, and review the possibility to build mutually beneficial economic relations. The main witnesses are the signed agreement on investment liberalization in 2018, the visit of Japanese Foreign Minister Taro Kono to Armenia for developing bilateral relations in the fields of banking, innovation, food security, information technology, artificial intelligence, cultural exchange, scientific and educational projects, as well as active contacts of Armenian President with Japanese organizations. 